Portland State University

PDXScholar

1992

\title{
An improved formulation of the temperature dependence of the Gummel-Poon bipolar transistor model equations
}

Chorng-Lii Liou

Portland State University

Follow this and additional works at: https://pdxscholar.library.pdx.edu/open_access_etds

Part of the Electrical and Computer Engineering Commons Let us know how access to this document benefits you.

Recommended Citation

Liou, Chorng-Lii, "An improved formulation of the temperature dependence of the Gummel-Poon bipolar transistor model equations" (1992). Dissertations and Theses. Paper 4361.

https://doi.org/10.15760/etd.6217

This Thesis is brought to you for free and open access. It has been accepted for inclusion in Dissertations and Theses by an authorized administrator of PDXScholar. Please contact us if we can make this document more accessible: pdxscholar@pdx.edu. 
AN ABSTRACT OF THE THESIS OF Chorng-Lii Liou for the Master of Science in Electrical and Computer Engineering presented May 7, 1992.

Title: An Improved Formulation of the Temperature Dependence of the Gummel-Poon Bipolar Transistor Model Equations.

APPROVED BY THE MEMBERS OF THE THESIS COMMITTEE:

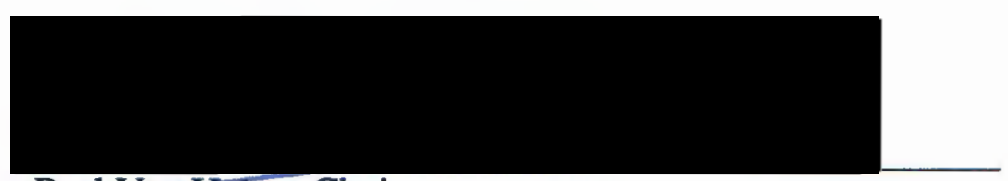

$$
\text { Paul Van Halen, Chair }
$$

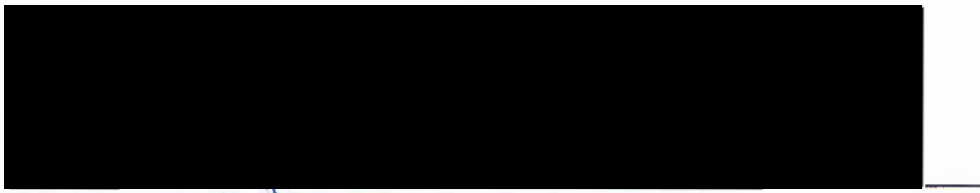

Malgorzata E. Chrzanowska-Jeske

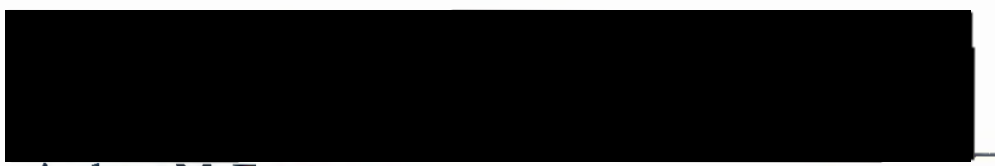

Andrew M. Fraser

A number of shortcomings were found after complete derivation of the temperature dependence of equations, and the expressions related to the Early effect in the present Gummel-Poon 2 model, as implemented in the TEKSPICE program. The formulation and application of improved model equations is presented, followed by a detailed comparison of the existing model with the one developed in this work. 
AN IMPROVED FORMULATION OF THE TEMPERATURE DEPENDENCE OF THE GUMMEL-POON BIPOLAR TRANSISTOR MODEL EQUATIONS

\author{
by \\ CHORNG-LII LIOU
}

A thesis submitted in partial fulfillment of the requirements for the degree of

\author{
MASTER OF SCIENCE \\ in \\ ELECTRICAL AND COMPUTER ENGINEERING
}

Portland State University

1992 
TO THE OFFICE OF GRADUATE STUDIES:

The members of the Committee approve the thesis of Chorng-Lii Liou presented May 7, 1992.

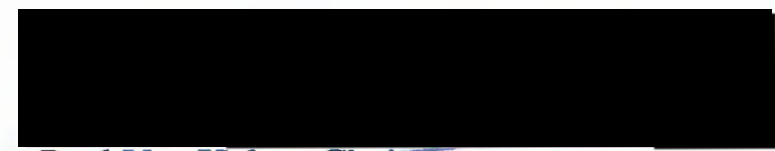

Paul Van Halen, Chair

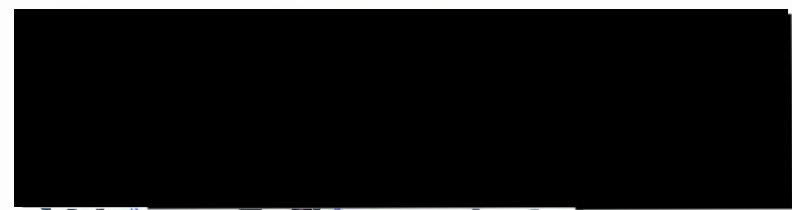

Malgorzata E. Chrzanowska-Jeske (1)

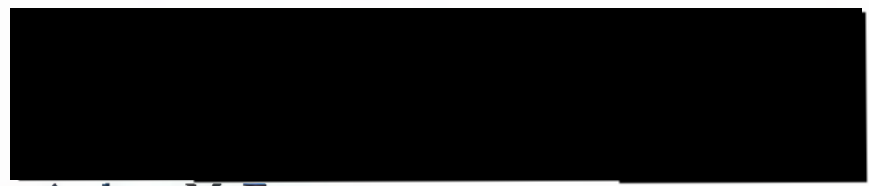

Andrew M. Fraser

\section{APPROVED:}

Rolf Schaumann, Chair, Department of Electrical Engineering

C. Wuilam Savery, Interim Vice Provost for Graduate Studies and Research 


\section{ACKNOWLEDGEMENTS}

I would like to express deep appreciation to Dr. Paul Van Halen for his sustaining guidance and support for this work and throughout my master's studies. Special thanks are due to Dr. Malgorzata Chrzanowska-Jeske and Dr. Andrew Fraser for their interest and support. My wife, Tsai-Hsing, deserves special thanks for her encouragement and help during my academic years. Finally, I am very glad to present my parents with this thesis and grateful to them for their endless support. 


\section{TABLE OF CONTENTS}

\section{PAGE}

ACKNOWLEDGEMENTS.................................................. iii

LIST OF FIGURES .................................................... v

\section{CHAPTER}

I INTRODUCTION ....................................... 1

II FORMULATION AND APPLICATION...................... 4

Junction Saturation Current................................ 4

Ideal Current Gain....................................... 9

Built-In Junction Potential............................... 14

Zero-Bias Junction Capacitance............................. 17

Leakage Saturation Current................................ 20

Charge-Control Model..................................... 25

Application......................................... 27

III DISCUSSION AND COMPARISON ........................ 35

Junction Saturation Current................................. 35

Ideal Current Gain....................................... 46

Built-In Junction Potential................................ 48

Zero-Bias Junction Capacitance............................ 50

Leakage Saturation Current................................ 56

Charge-Control Model.................................... 60

Comparison............................................. 61

IV CONCLUSION......................................... 69

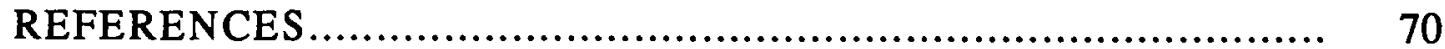




\section{LIST OF FIGURES}

1. Energy Gap as Function of Temperature for Two Different

Expressions....................................... 38

2. Comparison of Exponential Terms for Energy Gap................ 39

3. Summary of Bandgap Narrowing from Different

Measurements.......................................... 41

4. Comparison of the Simplified Equation with Effective-Mass

Ratio.

5. Comparison of the Simplified Equation with Electron-Mobility

Ratio

6. Comparison of the Simplified Equation with Hole-Mobility

Ratio.

7. Comparison of the Constant $\gamma_{T}^{C^{\infty}}$ with the Varied $\gamma_{T}^{\mathcal{C}^{\circ}}$ for the

Emitter-Base Junction..................................... 53

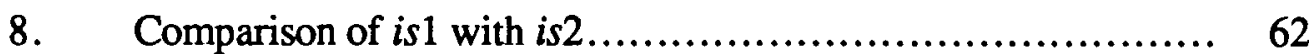

9. Comparison of an npn Transistor with a pnp Transistor

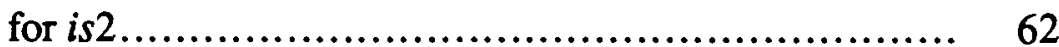

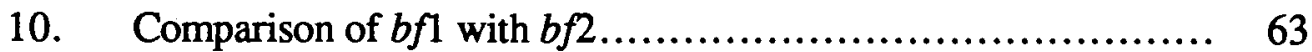

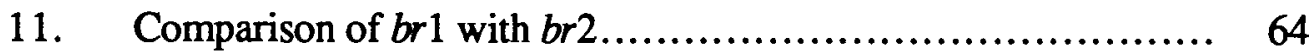

12. Comparison of an npn Transistor with a pnp Transistor

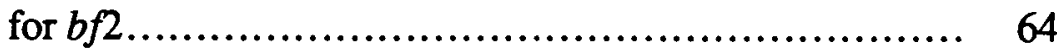

13. Comparison of an npn Transistor with a pnp Transistor

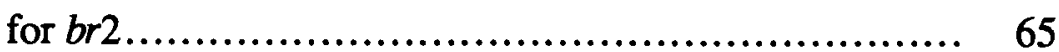

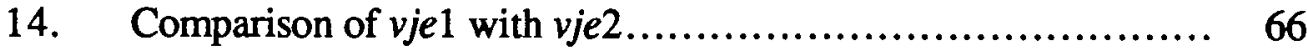


15. Comparison of vje1 with vje2 in GaAs........................... 66

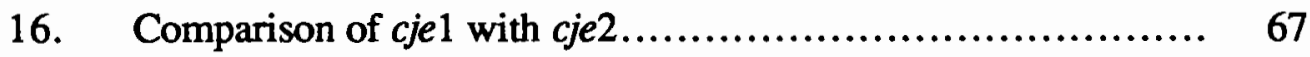

17. Comparison among ise 1 , ise 2 , and ise 2 with Theoretical

Lifetime........................................... 68 


\section{CHAPTER I}

\section{INTRODUCTION}

CAD (Computer-Aided Design) has been broadly used in various areas. For a circuit designer, the usefulness of CAD is well established (e.g. [1]). Observing waveforms and frequency responses of voltages and currents without loading the circuit as a probe would in an actual circuit, predicting the performances of an IC (Integrated Circuit) at high frequencies without the parasitics a breadboard introduces, and doing noise, sensitivity, worstcase and statistical analyses are some of the examples where CAD can be utilized.

The SPICE (Simulation Program with Integrated Circuit Emphasis) program has been used as an important computer-aid for the design of integrated circuits. The SPICE program provides a structure for a circuit simulation so that the behavior of a circuit, such as nonlinear DC (Direct Current), nonlinear transient, or linear small-signal AC (Alternating Current) analysis, can be performed. The basic, essential part of the SPICE program is its library of active-device models. Different models present different functions that can change the behavior of circuits. These models include the diode, bipolar junction transistor, MOSFET (Metal-Oxide-Semiconductor Field-Effect Transistor), and JFET (Junction Field-Effect Transistor). This paper focuses on the bipolar junction transistor model.

The fundamental theory of the bipolar junction transistor models is based on the EM (Ebers-Moll) model. The E-M model is a nonlinear and first-order DC model. By introducing the second-order effects, Gummel and Poon [2] developed the Gummel-Poon model. These second-order effects are:

1. The variation of current gain at low-current level;

2. The variation of current gain at high-current level; 
3. Basewidth modulation (Early effect);

4. The variation of transit time with collector current.

Since the non-ideal conditions have been considered and three effects (effect 2,3, and 4) are treated together, the Gummel-Poon model is the most accurate and complete among the existing models. The Gummel-Poon model has been implemented in the SPICE program in order to present the terminal characteristics of the bipolar junction transistors.

The Gummel-Poon model discussed in this paper is named GP2 model as implemented in the TEKSPICE program, developed by Boyle at Tektronix [3]. The GP2 model is described by a number of equations based on the physics of the transistor device and some special functions. Basically, this intricate program is built by some fundamental elements, such as physical constants, operating conditions, and model parameters [1]. Temperature is one of the operating conditions deciding the environment in which the analysis is to be performed. To predict transistor performances at a different temperature, the temperature effects for model parameters are included in the program. These specific temperature-related model parameters are represented by equations. Throughout these equations, the temperature behavior of a transistor can be performed.

The focus of this paper is on discussing the temperature effects of the specific parameters and the Early effect in the section of the charge-control model. All effects are described by equations. The purposes of this paper are to correct some shortcomings that were found in the present model and to obtain a more general, physical-meaning model based on related research. In order to obtain a better model, all equations based on the original definitions will be rederived. The rederived equations include some complex formulas. The simplified expressions instead of these complex formulas will be employed so that simpler rederived equations can be applied to the GP2 model. The process of the formation of the rederived equations and the application of the rederived equations will be presented in Chapter II. The rederived model is characterized by some added parameters, which will be discussed in Chapter III. Chapter III also contains a discussion of the drawbacks in the present model and a comparison of differences between the two models. 
Lastly, conclusion will be made in Chapter IV. 


\section{CHAPTER II}

\section{FORMULATION AND APPLICATION}

In this chapter, the equations of specific temperature-related parameters and expressions in the section of the charge-control model will be rederived according to their definitions. There are eleven specific temperature-related parameters and expressions for Early effect in the charge-control model to be derived in each of the following sections. In each section the definition of a specific parameter is, first of all, represented by a physical/ empirical expression as a function of temperature. Secondly, the derivative with respect to temperature of this parameter will be calculated. Finally, the derivative expression will be integrated with respect to temperature with the actual temperature and the nominal temperature as limits, so that parameter expression can be written as a function of the nominal temperature. The actual temperature can be in the $250 \mathrm{~K}$ to $500 \mathrm{~K}$ range. The temperature dependent parameters under study are the junction saturation current, ideal forward and reverse current gains, built-in junction potentials in emitter-base, basecollector, and collector-substrate junctions, zero-bias junction capacitances in emitter-base, base-collector, and collector-substrate junctions, and leakage saturation currents in emitterbase and base-collector junctions. All these parameters are derived under the conditions of one dimension and zero applied bias. Next, expressions for the Early effect will be modified. The last, the application of the equations for the temperature dependent parameters and the Early effect in the GP2 model will be discussed.

\section{JUNCTION SATURATION CURRENT}

For an active npn transistor, if no recombination is considered, the total current is [4]: 


$$
I_{n}=I_{s}\left[\exp \left(\frac{V_{B E}}{V_{t}}\right)-\exp \left(\frac{V_{B C}}{V_{t}}\right)\right]
$$

where

$I_{s}$ is the total saturation current and $V_{t}=\frac{K T}{q}$.

$$
I_{s}=\frac{q^{2} A_{E}^{2} n_{i}^{2} \tilde{D}_{n}}{Q_{B T}} \quad \text { and } \quad Q_{B T}=q A_{E} \int_{0}^{X_{B}} P(x) d x
$$

$Q_{B T}$ is total base charge and represented by bias dependent components.

The total base charge is:

$$
Q_{B T}=Q_{B 0}+Q_{E}+Q_{C}+Q_{F}+Q_{R}
$$

where

$Q_{B O}$ is the "built-in" total base charge and defined:

$$
Q_{B 0}=q A_{E} \int_{0}^{X_{B}} N_{A}(x) d x
$$

Early effect and high-current effect, the second-order effects, are represented by $Q_{E}, Q_{C}$, and $Q_{F}, Q_{R}$ respectively. $Q_{E}$ and $Q_{C}$ are emitter and collector chargestorage contributions. $Q_{F}$ and $Q_{R}$ are the charges associated with forward and reverse injection of base minority carriers at the high applied bias.

Let

$$
q_{b}=\frac{Q_{B T}}{Q_{B 0}}
$$

and substitution of this into the saturation current, $I_{s}$, gives

$$
I_{s}=\frac{q^{2} A_{E}^{2} n_{i}^{2} \widetilde{D}_{n}}{Q_{B 0} q_{b}}=\frac{I s}{q_{b}} \quad \text { where } \quad I s=\frac{q^{2} A_{E}^{2} n_{i}^{2} \widetilde{D}_{n}}{Q_{B 0}}
$$

Is is the "built-in" junction saturation current used in the Gummel-Poon model and influenced only by one of the operating conditions: temperature. Therefore, the definition 
of junction saturation current for an npn transistor is obtained.

\section{Definition}

$$
I s=\frac{q^{2} A_{E}^{2} n_{i e}^{2} \widetilde{D}_{n}}{Q_{B 0}}=\frac{q A_{E} n_{i e}^{2} \widetilde{D}_{n}}{\int_{0}^{X_{B}} N_{A}(x) d x}=\frac{q A_{E} n_{i e}^{2} \widetilde{D}_{n}}{N_{A}^{B}}
$$

where

$A_{E}$ is emitter area. $n_{i e}$ is effective intrinsic carrier concentration which havilydoped effect is included. $\widetilde{D}_{n}$ is average diffusion coefficient of minority carriers in the base and assumed very weak position dependent. $N_{A}^{B}$ is dopant concentrations in the base. The minority carriers in the base are electrons.

Both $n_{i e}$ and $\widetilde{D}_{n}$ are temperature dependent and will be discussed below.

The effective intrinsic carrier concentration, $n_{i e}$, is defined $[5,6]$ as follows,

$$
\begin{aligned}
n_{i e} & =2 \times\left(\frac{2 \pi m_{0} K}{h^{2}}\right)^{3 / 2} \times\left(\frac{m_{e}}{m_{0}} \frac{m_{h}}{m_{0}}\right)^{3 / 4} \times T^{3 / 2} \times \exp \left(-\frac{q E_{g}}{2 K T}\right) \\
& =2.509 \times 10^{19} \times\left(m_{c} m_{v}\right)^{3 / 4} \times\left(\frac{T}{300}\right)^{3 / 2} \times \exp \left(-\frac{q E_{g}}{2 K T}\right)
\end{aligned}
$$

where

$m_{c}$ is the effective electron mass. $m_{\nu}$ is the effective hole mass.

$E_{g}$ is energy gap including the havily-doped effect.

$m_{0}, K$, and $h$ are physical constants.

$m_{c}, m_{v}[6]$, and $E_{g}[7]$ are temperature dependent and shown as follows,

$$
\begin{aligned}
& m_{c}(T)=1.045+4.5 \times 10^{-4} T \\
& m_{\nu}(T)=0.523+1.4 \times 10^{-3} T-1.4 \times 10^{-6} T^{2}
\end{aligned}
$$




$$
E_{g}(T)=E G B-\alpha T
$$

where

$$
\begin{aligned}
& E G B=E G-\Delta E_{g} \\
& \Delta E_{g}=0.009 \times\left[\log _{e}\left(\frac{N}{N_{0}}\right)+\sqrt{\left[\log _{e}\left(\frac{N}{N_{0}}\right)\right]^{2}+0.5}\right]
\end{aligned}
$$

$E G$ is energy gap at $0 \mathrm{~K} . \Delta E_{g}[7]$ is bandgap narrowing because of havilydoped effect. $N$ is dopant concentrations. $N_{0}$ and $\alpha$ are constants. $\Delta E_{g}$ is assumed temperature independent.

The average diffusion coefficient of minority carriers, $\widetilde{D}_{n}$, is defined [4]:

$$
\widetilde{D}_{n}=\frac{K T}{q} \mu_{n}
$$

where

$\mu_{n}$ is mobility of minority carriers and temperature dependent.

The expression of majority-carrier mobilities as a function of temperature is used to demonstrate the temperature behavior of minority-carrier mobilities. This expression of majority-carrier mobilities for electrons is [8]:

$$
\mu_{n}(T)=\frac{88}{T_{n}^{0.57}}+\frac{\frac{7.4 \times 10^{8}}{T^{2.33}}}{1+\frac{0.88 N}{1.26 \times 10^{17} T_{n}^{2.546}}}
$$

where

$$
T_{n}=\frac{T}{300}
$$

Up to this section, all formulas which are related to temperature for the junction saturation current are obtained. Next, the derivative with respect to temperature of the junction saturation current, equation (2.1.1), will be calculated. 


$$
\begin{aligned}
\frac{\partial I s(T)}{\partial T} & =\frac{q A_{E}}{N_{A}^{B}} \times\left[\widetilde{D}_{n}(T) \times \frac{\partial n_{i e}^{2}(T)}{\partial T}+n_{i e}^{2}(T) \times \frac{\partial \widetilde{D}_{n}(T)}{\partial T}\right] \\
& =I s(T) \times\left[\frac{1}{n_{i e}^{2}(T)} \frac{\partial n_{i e}^{2}(T)}{\partial T}+\frac{1}{\widetilde{D}_{n}(T)} \frac{\partial \widetilde{D}_{n}(T)}{\partial T}\right]
\end{aligned}
$$

Integration

Equation (2.1.11) is integrated with Tnom and actual $T$ as limits, $I s(T)$ and Is(Tnom) are at the actual temperature $T$ and nominal temperature $T$ nom, respectively.

$$
\int_{I s(\text { Tnom })}^{I s(T)} \frac{d I S}{I s}=\int_{T n o m}^{T}\left[\frac{d n_{i e}^{2}(T)}{n_{i e}^{2}(T)}+\frac{d \widetilde{D}_{n}(T)}{\widetilde{D}_{n}(T)}\right]
$$

$I s(T)$ is obtained by solving (2.1.12).

$$
I s(T)=I s(\text { Tnom }) \times\left[\frac{n_{i e}^{2}(T) \widetilde{D}_{n}(T)}{n_{i e}^{2}(\text { Tnom }) \tilde{D}_{n}(\text { Tnom })}\right]
$$

Substitution of equations (2.1.2) and (2.1.8) into equation (2.1.13) gives

$$
\begin{aligned}
I s(T)=I s(\text { Tnom }) \times & {\left[\frac{m_{c}(T) m_{v}(T)}{m_{c}(\text { Tnom }) m_{v}(\text { Tnom })}\right]^{3 / 2} \times\left[\frac{T}{\text { Tnom }}\right]^{4} \times\left[\frac{\mu_{n}(T)}{\mu_{n}(\text { Tnom })}\right] \times } \\
& {\left[\frac{\exp \left(-\frac{q E_{g}(T)}{K T}\right)}{\exp \left(-\frac{q E_{g}(\text { Tnom })}{K \text { Tnom }}\right)}\right] }
\end{aligned}
$$

Replacement of equations (2.1.3-5) and (2.1.9-10) into (2.1.14) yields

$$
I s(T)=I s(\text { Tnom }) \times(M T)^{3 / 2} \times(\text { ratio })^{4} \times\left(U T_{n}\right) \times \exp \left(\frac{E G B}{V_{t}}(\text { ratio - 1) })\right.
$$

where

$$
\begin{aligned}
M T & =\left(\frac{m_{c}(T)}{m_{c}(\text { Tnom })}\right) \times\left(\frac{m_{\nu}(T)}{m_{\nu}(\text { Tnom })}\right)=M E T \times M H T \\
M E T & =(\text { ratio }) \times\left[1-\frac{1-(\text { ratio })^{-1}}{1+4.306 \times 10^{-4} \text { Tnom }}\right]
\end{aligned}
$$




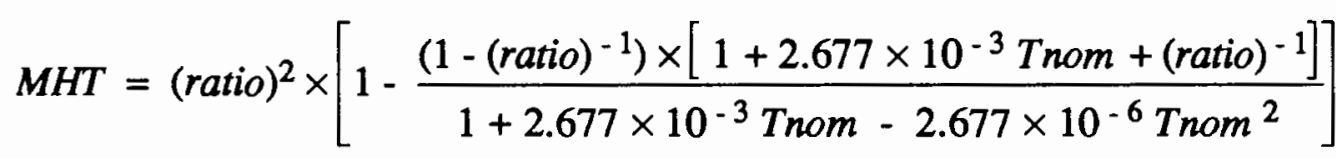

ratio $=\frac{T}{\text { Tnom }}$

$U T_{n}=\frac{\mu_{n}(T)}{\mu_{n}(\text { Tnom })}$

$=(\text { ratio })^{-0.57} \times\left\{1+\frac{\left[(\text { ratio })^{0.786} \times\left(\frac{\text { Tnom }^{2.546}+1.415 \times 10^{-11} N}{T^{2.546}+1.415 \times 10^{-11} N}\right)-1\right]}{\left[1+\frac{3.071 \times 10^{-6}\left(\text { Tnom }^{2.546}+1.415 \times 10^{-11} N\right)}{\text { Tnom }^{0.786}}\right]}\right\}$

$E G B=E G-\Delta E_{g}$ and $E G=1.206 \mathrm{eV}$ for silicon.

$V_{t}=\frac{K T}{q}$

The expression of the actual-temperature junction saturation current based on the nominal-temperature junction saturation current has been derived in equation (2.1.15).

\section{IDEAL CURRENT GAIN}

The current gain is the ratio of collector current to base current. In the GummelPoon model the ideal current gain is applied in the ideal base-current component, which is derived from the E-M model. The forward or reverse current gain, $\beta_{F, R}$, is defined as follows [4],

$$
\beta_{F, R}=\frac{\alpha_{F, R}}{1-\alpha_{F, R}} \quad \text { and } \quad \alpha_{F, R}=\gamma \alpha_{T}
$$

where

$\gamma$ is the emitter efficiency and $\alpha_{T}$ is the transport factor. 
If no recombination in the base is assumed, the transport factor is equal to one. Thus the current gain is only controlled by emitter efficiency. For an npn transistor, it equals:

$$
\beta_{F, R} \approx \frac{\gamma_{F, R}}{1-\gamma_{F, R}} \quad \text { and } \quad \gamma_{F, R}=\frac{1}{1+\frac{I_{p}}{I_{n}}}
$$

Substitution of $\gamma_{F, R}$ into $\beta_{F, R}$ gives

$$
\beta_{F, R}=\frac{I_{n}}{I_{p}}
$$

where

$I_{n}$ is the eletron current injected into the base and $I_{p}$ is the hole current which flows into the emitter or collector.

According to the definitions of $I_{n}$ and $I_{p}$ [9], the current gain is:

and

$$
\beta_{F, R}=\frac{I_{n}}{I_{p}}=\frac{I_{s}\left[\exp \left(\frac{q V_{B E, B C}}{K T}\right)-1\right]}{I_{d}\left[\exp \left(\frac{q V_{B E, B C}}{K T}\right)-1\right]}=\frac{I_{s}}{I_{d}}
$$

$$
I_{s}=\frac{q^{2} A_{E, C}^{2} n_{i e B}^{2} \tilde{D}_{n}}{Q_{B T}} \quad \text { and } \quad I_{d}=\frac{q^{2} A_{E, C}^{2} n_{i e E, C}^{2} \tilde{D}_{p}}{Q_{E T, C T}}
$$

where

$A_{E, C}$ is the area for emitter or collector, $n_{i \in B}^{2}$ is the effective intrinsic concentration in the base. $n_{i e E, C}^{2}$ is the effective intrinsic concentration in the emitter or collector, $Q_{B T}$ is the total base charge and $Q_{E T, C T}$ is total emitter or collector charge. These total charges are obtained under the intermediate-voltage level. $\widetilde{D}_{n}$ is the diffusion coefficient for electron and $\widetilde{D}_{p}$ is the diffusion coefficient for hole. 
Substitution of $I_{s}$ and $I_{d}$ into $\beta_{F, R}$ gives

$\beta_{F, R}=\frac{n_{i e B}^{2} \widetilde{D}_{n} Q_{E T, C T}}{n_{i e E, C}^{2} \widetilde{D}_{p} Q_{B T}}$

The ideal current gain for forward or reverse is obtained.

\section{Definition}

The forward current gain is defined as follows,

$$
\beta_{F}=\frac{n_{i e B}^{2} \widetilde{D}_{n} Q_{E T}}{n_{i e E}^{2} \widetilde{D}_{p} Q_{B T}}
$$

Using the definition of effective intrinsic concentration and diffusion coefficient, equations (2.1.2) and (2.1.8), equation (2.2.1) becomes:

$$
\begin{aligned}
\beta_{F}(T) & =\frac{\mu_{n}(T) \times Q_{E T} \times \exp \left(-\frac{q E_{g B}(T)}{K T}\right)}{\mu_{p}(T) \times Q_{B T} \times \exp \left(-\frac{q E_{g E}(T)}{K T}\right)} \\
& =\left(\frac{Q_{E T}}{Q_{B T}}\right) \times\left(\frac{\mu_{n}(T)}{\mu_{p}(T)}\right) \times \exp \left(\frac{q \Delta E G E}{K T}\right)
\end{aligned}
$$

where

$Q_{E T}$ and $Q_{B T}$ are only dependent on bias.

$\mu_{n}$ and $\mu_{p}$ are mobilities of minority carriers and temperature dependent. The expression of majority-carrier mobilities as a function of temperature is used to demonstrate the temperature behavior of minority-carrier mobilities. The expression of majority-carrier mobilities for electrons in the base is defined in equation (2.1.9) and the expression for holes in the emitter is as follows [8]:

$$
\mu_{p}(T)=\frac{54.3}{T_{n}^{0.57}}+\frac{\frac{1.36 \times 10^{8}}{T^{2.23}}}{1+\frac{0.88 N}{2.35 \times 10^{17} T_{n}^{2.546}}}
$$


$\triangle E G E$ is the difference between the bandgap narrowing in emitter and in base. It is temperature independent and is equal to:

$$
\Delta E G E=E G E-E G B=\Delta E_{g E}-\Delta E_{g B}
$$

The reverse current is obtained by substituting the notations of collector into emitter's.

$$
\beta_{R}=\left(\frac{Q_{C T}}{Q_{B T}}\right) \times\left(\frac{\mu_{n}(T)}{\mu_{p}(T)}\right) \times \exp \left(\frac{q \Delta E G C}{K T}\right)
$$

where

$\mu_{p}(T)$ is the hole mobilities in the collector.

$$
\Delta E G C=E G C-E G B=\Delta E_{g C}-\Delta E_{g B}
$$

$\triangle E G C$ is the difference between the bandgap narrowing in collector and in base.

The current gains as a function of temperature have been defined.

\section{Forward current gain}

Taking the derivative with respect to temperature of equation (2.2.2) gives

$$
\begin{aligned}
& \frac{\partial \beta_{F}(T)}{\partial T}=\left(\frac{Q_{E T}}{Q_{B T}}\right) \times\left(\frac{\mu_{n}(T)}{\mu_{p}(T)}\right) \times \exp \left(\frac{q \Delta E G E}{K T}\right) \\
& \times\left[\frac{1}{\mu_{n}} \frac{\partial \mu_{n}(T)}{\partial T}-\frac{q \Delta E G E}{K T^{2}}-\frac{1}{\mu_{p}} \frac{\partial \mu_{p}(T)}{\partial T}\right] \\
& \frac{\partial \beta_{F}(T)}{\partial T}=\beta_{F}(T) \times\left[\frac{1}{\mu_{n}(T)} \frac{\partial \mu_{n}(T)}{\partial T}-\frac{q \Delta E G E}{K T^{2}}-\frac{1}{\mu_{p}(T)} \frac{\partial \mu_{p}(T)}{\partial T}\right]
\end{aligned}
$$

Calculating the definite integral with $\beta_{F}($ Tnom $)$ and $\beta_{F}(T)$ as limits gives 


$$
\begin{gathered}
\int_{\beta_{F}(T n o m)}^{\beta_{F}(T)} \frac{d \beta_{F}(T)}{\beta_{F}(T)}=\int_{\mu_{n}(T n o m)}^{\mu_{n}(T)} \frac{d \mu_{n}(T)}{\mu_{n}(T)}-\int_{T n o m}^{T} \frac{q \Delta E G E}{K T^{2}} d T \\
-\int_{\mu_{p}(T \text { nom })}^{\mu_{p}(T)} \frac{d \mu_{p}(T)}{\mu_{p}(T)}
\end{gathered}
$$

$\beta_{F}(T)$ is obtained by solving equation (2.2.8).

$$
\begin{aligned}
\beta_{F}(T)= & \beta_{F}(\text { Tnom }) \times\left(\frac{\mu_{n}(T)}{\mu_{n}(\text { Tnom })}\right) \times\left(\frac{\mu_{p}(T)}{\mu_{p}(\text { Tnom })}\right)^{-1} \\
& \times \exp \left(\frac{q \Delta E G E}{K}\left(\frac{1}{T}-\frac{1}{\text { Tnom }}\right)\right) \\
= & \beta_{F}(\text { Tnom }) \times\left(U T_{n}\right) \times\left(U T_{p}\right)^{-1} \times \exp \left(\frac{\Delta E G E}{V_{t}}(1-\text { ratio })\right)
\end{aligned}
$$

where

$U T_{n}$ is defined in equation (2.1.20). $U T_{p}$ is defined as follows,

$$
U T_{p}=(\text { ratio })^{-0.57} \times\left\{1+\frac{\left[(\text { ratio })^{0.886}\left(\frac{\text { Tnom }^{2.546}+7.589 \times 10^{-12} N}{T^{2.546}+7.589 \times 10^{-12} N}\right)-1\right]}{\left[1+\frac{1.895 \times 10^{-6}\left(\text { Tnom }^{2.546}+7.589 \times 10^{-12} N\right)}{\text { Tnom }^{0.886}}\right]}\right\}
$$

The expression of the actual-temperature forward current gain based on the nominal-temperature forward current gain has been derived in equation (2.2.9).

\section{$\underline{\text { Reverse current gain }}$}

The reverse current gain can be obtained by using the same procedures described in the section of the forward current gain. The expression for the reverse current gain is:

$$
\beta_{R}(T)=\beta_{R}(\text { Tnom }) \times\left(U T_{n}\right) \times\left(U T_{p}\right)^{-1} \times \exp \left(\frac{\Delta E G C}{V_{t}}(1-\text { ratio })\right)
$$


where

$U T_{n}$ and $U T_{p}$ are defined in equations (2.1.20) and (2.2.10).

$\triangle E G C$ is defined in (2.2.6).

\section{BUILT-IN JUNCTION POTENTIAL}

When p-type and n-type semiconductors are brought into contact, the electron current and hole current will diffuse into opposite sides and, at the same time, the electric field is built opposing the flow of the currents. This built-in electric field causes a built-in potential barrier between the p-n junction. With the assumptions of the depletion approximation and the very small carrier concentration in the space-charge region, the built-in potential can be obtained by solving Poisson's equation. This built-in junction potential is the total potential change in the space-charge region from the edge of the neutral n-type region to the edge of the neutral p-type region. The well-known equation for built-in junction potential is defined in equation (2.3.1).

$$
\Phi_{i}(T)=\frac{K T}{q} \times \log _{e}\left(\frac{N_{a} N_{d}}{n_{i}^{2}(T)}\right)
$$

where

$N_{a}$ and $N_{d}$ are impurity concentrations of p-type and n-type materials respectively. $n_{i}$ is the intrinsic carrier concentration in the space-charge region. The intrinsic carrier concentration is temperature dependent and defined in equation (2.1.2), which is:

$$
n_{i}=2.5 \times 10^{19} \times\left(m_{c}\right)^{3 / 4} \times\left(m_{\nu}\right)^{3 / 4} \times\left(\frac{T}{300}\right)^{3 / 2} \times \exp \left(-\frac{q E_{g}}{2 K T}\right)
$$

where no bandgap narrowing is included in the expression of energy gap, i.e., $E G=E G B$ in equation (2.1.6).

The built-in junction potential as a function of temperature is obtained.

Taking the derivative with respect to temperature of equation (2.3.1) gives 


$$
\begin{aligned}
\frac{\partial \Phi_{i}(T)}{\partial T} & =\frac{K T}{q} \times \log _{e}\left(\frac{N_{a} N_{d}}{n_{i}{ }^{2}(T)}\right) \times\left[\frac{1}{T}-\frac{\frac{1}{n_{i}{ }^{2}(T)} \frac{\partial n_{i}{ }^{2}(T)}{\partial T}}{\log _{e}\left(\frac{N_{a} N_{d}}{n_{i}{ }^{2}(T)}\right)}\right] \\
& =\Phi_{i}(T) \times\left[\frac{\log _{e}\left(N_{a} N_{d}\right)-\log _{e}\left(n_{i}{ }^{2}(T)\right)-\frac{T}{n_{i}{ }^{2}(T)} \frac{\partial n_{i}{ }^{2}(T)}{\partial T}}{T \log _{e}\left(N_{a} N_{d}\right)-T \log _{e}\left(n_{i}{ }^{2}(T)\right)}\right]
\end{aligned}
$$

Calculating the definite integral with $\Phi_{i}(T)$ and $\Phi_{i}($ Tnom $)$ as limits gives

$$
\begin{aligned}
\int_{\Phi_{i}(\text { Tnom })}^{\Phi_{i}(T)} \frac{d \Phi_{i}}{\Phi_{i}} & =\int_{T \text { nom }}^{T}\left[\frac{\log _{e}\left(N_{a} N_{d}\right)-\log _{e}\left(n_{i}{ }^{2}(T)\right)-\frac{T}{n_{i}^{2}} \frac{d n_{i}{ }^{2}(T)}{d T}}{T \log _{e}\left(N_{a} N_{d}\right)-T \log _{e}\left(n_{i}{ }^{2}(T)\right)}\right] d T \\
& =\int_{\text {Tnom }}^{T}\left[\frac{d\left[T \log _{e}\left(N_{a} N_{d}\right)-T \log _{e}\left(n_{i}^{2}(T)\right)\right]}{T \log _{e}\left(N_{a} N_{d}\right)-T \log _{e}\left(n_{i}{ }^{2}(T)\right)}\right]
\end{aligned}
$$

By solving the integration, equation (2.3.4) becomes:

$$
\begin{gathered}
\log _{e}\left(\frac{\Phi_{i}(T)}{\Phi_{i}(\text { Tnom })}\right)=\log _{e}\left(\frac{T \log _{e}\left(N_{a} N_{d}\right)-T \log _{e}\left(n_{i}{ }^{2}(T)\right)}{\operatorname{Tnom} \log _{e}\left(N_{a} N_{d}\right)-\operatorname{Tnom} \log _{e}\left(n_{i}{ }^{2}(\text { Tnom })\right)}\right) \\
\frac{\Phi_{i}(T)}{\Phi_{i}(\text { Tnom })}=\frac{T}{\text { Tnom }}-\frac{T\left[\log _{e}\left(n_{i}{ }^{2}(T)\right)-\log _{e}\left(n_{i}{ }^{2}(\text { Tnom })\right)\right]}{\text { Tnom } \log _{e}\left(N_{a} N_{d}\right)-\text { Tnom } \log _{e}\left(n_{i}{ }^{2}(\text { Tnom })\right)} \\
=\frac{T}{\text { Tnom }}-\frac{\frac{K T}{q} \times \log _{e}\left(\frac{n_{i}{ }^{2}(T)}{n_{i}{ }^{2}(\text { Tnom })}\right)}{\Phi_{i}(\text { Tnom })}
\end{gathered}
$$

then

$$
\Phi_{i}(T)=\frac{T}{\text { Tnom }} \times \Phi_{i}(\text { Tnom })-\frac{K T}{q} \times \log _{e}\left(\frac{n_{i}{ }^{2}(T)}{n_{i}{ }^{2}(\text { Tnom })}\right)
$$


Substitution equations (2.3.2), (2.1.16), and (2.1.5) into (2.3.5) gives

$$
\Phi_{i}(T)=\Phi_{i}(\text { Tnom }) \times(\text { ratio })-\frac{3}{2} V_{t} \times \log _{e}\left(M T \times(\text { ratio })^{2}\right)+E G(1-\text { ratio })
$$

where

$V_{t}=\frac{K T}{q}, M T$ is defined in equation (2.1.16-18).

$E G$ is the energy gap at $0 \mathrm{~K}$.

The expression of the actual-temperature built-in junction potential based on the nominal-temperature built-in junction potential has been derived in equation (2.3.6). This expression will be applied to three junctions which are emitter-base, base-collector, and collector-substrate junctions. They are shown below.

Emitter-base junction

$$
v j e=V J E \times \text { ratio }- \text { vref }
$$

where

$$
\begin{aligned}
& \text { vje }=\Phi_{i}(T), V J E=\Phi_{i}(\text { Tnom }) \\
& \text { vref }=\frac{3}{2} V_{t} \times \log _{e}\left(M T \times(\text { ratio })^{2}\right)-E G(1-\text { ratio })
\end{aligned}
$$

Base-collector junction

$$
v j c=V J C \times \text { ratio }- \text { vref }
$$

where

$$
v j c=\Phi_{i}(T), V J C=\Phi_{i}(\text { Tnom })
$$

vref is defined in equation (2.3.9).

\section{Collector-substrate junction}

$$
v j s=V J S \times \text { ratio }- \text { vref }
$$

where 
$v j s=\Phi_{i}(T), V J S=\Phi_{i}($ Tnom $)$

$v r e f$ is defined in equation (2.3.9).

\section{ZERO-BIAS JUNCTION CAPACITANCE}

In the previous section, the built-in electric field and potential were presented. When two types of semiconductor make contact, there is a maximum electric field located at the interface. This field is caused by the stored space charge on the basis of Gauss' law. The amount of the space charge is the same on the both sides of the junction where a capacitive behavior is shown under small-signal AC conditions. This capacitive behavior is represented by $C$ (capacitance), the ratio of a differential space charge to differential voltage. It is:

$$
C_{j}=\frac{d Q}{d V}
$$

Capacitance can also be represented by the simple relationship for an arbitrarily doped junction and it is as follows [4],

$$
C_{j}=\frac{A \varepsilon}{x_{d}}
$$

where

$A$ is area. $\varepsilon$ is the dielectric constant of the semiconductor. $x_{d}$ is the width of the space-charge region.

By the definition of space-charge region [10], the capacitance can be written,

$$
C_{j}=\frac{\varepsilon A}{K_{c} V^{m}}=\frac{\varepsilon A}{K_{c} \varepsilon^{m}\left(\Phi_{i}-V_{A}\right)^{m}}=\frac{\varepsilon^{1-m} A}{K_{c}^{\prime} \Phi_{i}^{m}\left(1-\frac{V_{A}}{\Phi_{i}}\right)^{m}}=\frac{C_{j 0}}{\left(1-\frac{V_{A}}{\Phi_{i}}\right)^{m}}
$$

where

$C_{j}=C_{j 0}$ when the applied bias $V_{A}$ is zero. $C_{j 0}$ is the zero-bias junction capacitance or built-in junction capacitance and it is: 
$C_{j 0}=\frac{\varepsilon^{1-m} A}{K_{c}^{\prime} \Phi_{i}^{m}}$

where

$\Phi_{i}$ is built-in junction potential. $K_{c}^{\prime}$ represents each of the three types of junctions, they are:

$$
\begin{aligned}
K_{\mathrm{c}}^{\prime} & =\left(\frac{4}{q N}\right)^{m}, \quad m=\frac{1}{2} \text { for the symmetrical abrupt junction. } \\
& =\left(\frac{2}{q N}\right)^{m}, \quad m=\frac{1}{2} \text { for the one-sided abrupt junction. } \\
& =\left(\frac{12}{q a}\right)^{m}, \quad m=\frac{1}{3} \text { for the linearly graded junction. }
\end{aligned}
$$

$m$ is the junction graded coefficient.

The temperature dependent parameters for the zero-bias junction capacitance are the dielectric constant [11] and the built-in junction potential. Thus, the equation as a function of temperature for zero-bias junction capacitance is:

$$
C_{j 0}(T)=\frac{A \varepsilon^{1-m}(T)}{K_{c}^{\prime} \Phi_{i}^{m}(T)}
$$

where

$$
\varepsilon(T)=\frac{\delta}{4 \pi} \exp (p T)
$$

$\delta=1.2711 \times 10^{-9}$ and $p=7.8 \times 10^{-5}$ for silicon.

$\delta=1.7153 \times 10^{-9}$ and $p=1.38 \times 10^{-4}$ for germanium.

$\Phi_{i}$ is defined in (2.3.1) and $\Phi_{i}(T)=\frac{K T}{q} \times \log _{e}\left(\frac{N_{a} N_{d}}{n_{i}{ }^{2}(T)}\right)$

Taking the derivative with respect to temperature of equation (2.4.1) gives

$$
\frac{\partial C_{j 0}(T)}{\partial T}=\frac{A \varepsilon^{1-m}(T)}{K_{c}^{\prime} \Phi_{i}^{m}(T)} \times\left[\frac{1-m}{\varepsilon(T)} \frac{\partial \varepsilon(T)}{\partial T}-\frac{m}{\Phi_{i}(T)} \frac{\partial \Phi_{i}(T)}{\partial T}\right]
$$




$$
=C_{j 0}(T) \times\left[\frac{1-m}{\varepsilon(T)} \frac{\partial a(T)}{\partial T}-\frac{m}{\Phi_{i}(T)} \frac{\partial \Phi_{i}(T)}{\partial T}\right]
$$

Calculating the definite integral with $C_{j 0}($ Tnom $)$ and $C_{j 0}(T)$ as limits gives

$$
\int_{C_{j}(\text { Tnom })}^{C_{j 0(T)}} \frac{d C_{j 0}}{C_{j 0}}=\int_{\varepsilon(\text { Tnom })}^{\varepsilon(T)} \frac{(1-m) d \varepsilon}{\varepsilon}-\int_{\Phi_{i}(\text { Tnom })}^{\Phi_{i}(T)} \frac{m d \Phi_{i}}{\Phi_{i}}
$$

By solving the integration, equation (2.4.5) becomes:

$$
\begin{aligned}
& \log _{e}\left(\frac{C_{j 0}(T)}{C_{j 0}(\text { Tnom })}\right)=(1-m) \times \log _{e}\left(\frac{\varepsilon(T)}{\varepsilon(\text { Tnom })}\right)-m \times \log _{e}\left(\frac{\Phi_{i}(T)}{\Phi_{i}(\text { Tnom })}\right) \\
& \frac{C_{j 0}(T)}{C_{j 0}(\text { Tnom })}=\left(\frac{\varepsilon(T)}{\varepsilon(\text { Tnom })}\right)^{1-m} \times\left(\frac{\Phi_{i}(T)}{\Phi_{i}(\text { Tnom })}\right)^{-m}
\end{aligned}
$$

Replacement of equations (2.4.2) into (2.4.6) gives

$$
C_{j 0}(T)=C_{j 0}(\text { Tnom }) \times \exp (p \times(1-m) \times(T-T \text { nom })) \times\left(\frac{\Phi_{i}(T)}{\Phi_{i}(\text { Tnom })}\right)^{-m}
$$

where

$m$ is dependent on which doped junction is used.

$$
\frac{\Phi_{i}(T)}{\Phi_{i}(\text { Tnom })} \text { has been derived in equation (2.3.6) in last section. }
$$

The expression of the actual-temperature zero-bias junction capacitance based on the nominal-temperature zero-bias junction capacitance has been derived in equation (2.4.7). This expression will be applied to three junctions which are emitter-base, basecollector, and collector-substrate junctions. They are shown as follows. 
Emitter-base junction

$$
c j e=C J E \times \exp (p \times(1-M J E) \times(T-T \text { nom })) \times\left(\frac{v j e}{V J E}\right)^{-M J E}
$$

where

$$
\text { cje }=\Phi_{i}(T), C J E=\Phi_{i}(\text { Tnom })
$$

vje and $V J E$ are defined in equations (2.3.7-8).

$M J E$ is the emitter-base junction grading coefficient.

Base-collector junction

$$
c j c=C J C \times \exp (p \times(1-M J C) \times(T-T n o m)) \times\left(\frac{v j c}{V J C}\right)^{-M J C}
$$

where

$$
c j c=\Phi_{i}(T), C J C=\Phi_{i}(\text { Tnom })
$$

$v j c$ and $V J C$ are defined in equations (2.3.10-11).

$M J C$ is the base-collector junction grading coefficient.

Collector-substrate junction

$$
c j s=C J S \times \exp (p \times(1-M J S) \times(T-T \text { nom })) \times\left(\frac{v j S}{V J S}\right)^{-M J S}
$$

where

$$
c j s=\Phi_{i}(T), C J S=\Phi_{i}(\text { Tnom })
$$

$v j s$ and $V J S$ are defined in equations (2.3.12-13).

$M J S$ is the collector-substrate junction grading coefficient.

\section{LEAKAGE SATURATION CURRENT}

In the Gummel-Poon model the base current is defined in terms of a superposition 
of ideal and nonideal-diode components [4]. In the active region of operation, the base current is dominated by the ideal-component current. Since the nonideal-component current is comparable to the ideal-component current at low bias [12], the base current is dominated by the nonideal-component current. The nonideal current results from a combination of space-charge-region recombination, surface recombination, surfacechannel recombination [13]. With careful processing, the recombination currents in the surface and surface-channel can be made very small [1] and the nonideal component can be simply represented by the recombination current in the space-charge region. This nonideal current is defined as follows [13],

$$
I_{\text {nonideal }}=I_{0}\left[\exp \left(\frac{V_{A}}{\operatorname{nV}}\right)-1\right]
$$

where

$I_{0}$ is the leakage saturation current.

$n$ is the low current leakage emission coefficient.

$V_{A}$ is applied voltage.

The leakage saturation current is the current at zero bias and determined by the emission coefficient. It is defined as follows [14],

$$
I_{0}=\left[\frac{q n_{i} W_{S C R}}{2 \tau_{0}}\right]^{2 / n}
$$

where

$n_{i}$ is intrinsic carrier concentration without the effect of havily-doped.

$W_{S C R}$ is the width of the space-charge region.

$\tau_{0}$ is lifetime where $\tau_{0}=\tau_{n}=\tau_{p}$ is applied in the low-level injection, and $\tau_{n}$ and

$\tau_{p}$ are the lifetimes of the electron- and hole- excess carriers in the space-charge region.

Intrinsic carrier concentration, the width of the space-charge region, and lifetime are temperature dependent. Thus, the leakage saturation current is defined as follows, 


$$
I_{0}(T)=\left[\frac{q n_{i}(T) W_{S C R}(T)}{2 \tau_{0}(T)}\right]^{2 / n}
$$

where

$n_{i}(T)$ is defined in equation (2.3.2) and is shown:

$$
\begin{aligned}
n_{i}(T)=2.5 \times 10^{19} \times( & \left.m_{c}(T)\right)^{3 / 4} \times\left(m_{v}(T)\right)^{3 / 4} \times\left(\frac{T}{300}\right)^{3 / 2} \\
& \times \exp \left(-\frac{q E_{g}(T)}{2 K T}\right)
\end{aligned}
$$

$W_{S C R}(T)=x_{d}(T)=K_{c}^{\prime} \varepsilon^{m}(T) \Phi_{i}^{m}(T)$

where $x_{d}, K_{c}^{\prime}, \varepsilon$, and $\Phi_{i}$ are defined in the previous sections.

Lifetime is not only temperature dependent and also dopant concentration dependent. It can be represented by Shockley-Read-Hall lifetime and is shown as follows $[15]$,

$$
\tau^{S R H}(N, T)=\frac{\tau_{0}(T)}{1+\frac{N}{N_{0}}}
$$

where

$N$ is dopant concentration and $N_{0}$ is a constant.

Taking the derivative with respect to temperature of equation (2.5.1) gives

$$
\begin{aligned}
\frac{\partial I_{0}(T)}{\partial T}=\frac{2}{n} \times \frac{q n_{i}(T) W_{S C R}(T)}{\tau^{S R H}(T)} \times\left[\frac{1}{n_{i}(T)} \frac{\partial n_{i}(T)}{\partial T}\right. & \\
& \left.+\frac{1}{W_{S C R}(T)} \frac{\partial W_{S C R}(T)}{\partial T}-\frac{1}{\tau_{0}(T)} \frac{\partial \tau_{0}(T)}{\partial T}\right] \\
=\frac{2}{n} \times I_{0}(T) & {\left[\frac{1}{n_{i}(T)} \frac{\partial n_{i}(T)}{\partial T}+\frac{1}{W_{S C R}(T)} \frac{\partial W_{S C R}(T)}{\partial T}\right.} \\
& \left.-\frac{1}{\tau_{0}(T)} \frac{\partial \tau_{0}(T)}{\partial T}\right]
\end{aligned}
$$


23

Calculating the definite integral with $I_{0}(T)$ and $I_{0}$ (Tnom) as limits gives

$$
\begin{aligned}
\int_{I_{0}(\text { Tnom })}^{I_{0}(T)} \frac{n d I_{0}(T)}{2 I_{0}(T)}=\int_{n_{i}(T \text { nom })}^{n_{i}(T)} \frac{d n_{i}(T)}{n_{i}(T)} & +\int_{W_{S C R}(T \text { nom })}^{W_{S C R}(T)} \frac{d W_{S C R}(T)}{W_{S C R}(T)} \\
& -\int_{\tau_{0}(\text { Tnom })}^{\tau_{0}(T)} \frac{d \tau_{0}(T)}{\tau_{0}(T)}
\end{aligned}
$$

$I_{0}(T)$ is obtained by solving equation (2.5.6).

$$
\begin{aligned}
& \frac{n}{2} \log _{e}\left(\frac{I_{0}(T)}{I_{0}(\text { Tnom })}\right)=\log _{e}\left(\frac{n_{i}(T)}{n_{i}(\text { Tnom })}\right)+\log _{e}\left(\frac{W_{S C R}(T)}{W_{S C R}(\text { Tnom })}\right)-\log _{e}\left(\frac{\tau_{0}(T)}{\tau_{0}(\text { Tnom })}\right) \\
& {\left[\frac{I_{0}(T)}{I_{0}(\text { Tnom })}\right]^{n / 2}=\frac{n_{i}(T)}{n_{i}(\text { Tnom })} \times \frac{W_{S C R}(T)}{W_{S C R}(\text { Tnom })} \times\left(\frac{\tau_{0}(T)}{\tau_{0}(\text { Tnom })}\right)^{-1}}
\end{aligned}
$$

Substitution of equations (2.5.2) and (2.5.3) into (2.5.7) gives the first two terms of the right-hand side of (2.5.7) and they are as follows,

$$
\begin{aligned}
& \frac{n_{i}(T)}{n_{i}(\text { Tnom })}=(M T)^{3 / 4} \times\left(\frac{T}{\text { Tnom }}\right)^{3 / 2} \times \exp \left(\frac{E G}{2 V_{t}}(\text { ratio - } 1)\right) \\
& \frac{W_{S C R}(T)}{W_{S C R}(\text { Tnom })}=\left[\frac{\varepsilon(T)}{\varepsilon(\text { Tnom })} \times \frac{\Phi_{i}(T)}{\Phi_{i}(\text { Tnom })}\right]^{m}
\end{aligned}
$$

Equation (2.5.9) can be rewritten by referring equation (2.4.6)

$$
\begin{aligned}
\frac{W_{S C R}(T)}{W_{S C R}(\text { Tnom })} & =\frac{\varepsilon(T)}{\varepsilon(\text { Tnom })} \times \frac{C_{j 0}(\text { Tnom })}{C_{j 0}(T)} \\
& =\frac{C_{j 0}(\text { Tnom })}{C_{j 0}(T)} \times \exp (p \times(T-\text { Tnom }))
\end{aligned}
$$

Replacement of equations (2.5.8) and (2.5.10) into (2.5.7) gives

$$
\begin{gathered}
\frac{I_{0}(T)}{I_{0}(\text { Tnom })}=M T^{3 / 2 n} \times \text { ratio }^{3 / n} \times \exp \left(\frac{E G}{n V_{t}}(\text { ratio }-1)\right) \times\left[\frac{C_{j 0}(\text { Tnom })}{C_{j 0}(T)}\right]^{2 / n} \\
\quad \times \exp \left(p \times \frac{2}{n} \times(T-\text { Tnom })\right) \times\left(\frac{\tau_{0}(T)}{\tau_{0}(\text { Tnom })}\right)^{-2 / n}
\end{gathered}
$$


where

$M T$ is defined in equation (2.1.16-18). $E G$ is defined in equation (2.1.21).

$\frac{C_{j 0}(\text { Tnom })}{C_{j 0}(T)}$ is defined in equation (2.4.7).

$\frac{\tau_{0}(T)}{\tau_{0}(\text { Tnom })}$ is obtained by measurement.

The expression of the actual-temperature leakage saturation current based on the nominal-temperature leakage saturation current has been derived in equation (2.5.11). This expression will be applied to the two junctions, emitter-base and base-collector. They are shown below.

Emitter-basejunction

$$
\begin{aligned}
\text { ise }=I S E \times & M T^{3 / 2 N E} \times \text { ratio }^{3 / N E} \times \exp \left(\frac{E G}{N E V_{t}}(\text { ratio }-1)\right) \times\left[\frac{C J E}{c j e}\right]^{2 / N E} \\
& \times \tau \text { nalE }-2 / N E \times \exp \left(p \times \frac{2}{N E} \times(T-\text { Tnom })\right)
\end{aligned}
$$

where

ise $=I_{0}(T), I S E=I_{0}($ Tnom $)$

cje and $C J E$ are defined in equation (2.4.9).

$\tau$ nalE is the nomalized lifetime of the emitter-base junction.

$N E$ is the emission coefficient of the emitter-base junction.

Base-collector junction

$$
\begin{aligned}
\text { isC }=I S C \times & M T^{3 / 2 N C} \times \text { ratio }^{3 / N C} \times \exp \left(\frac{E G}{N C V_{t}}(\text { ratio }-1)\right) \times\left[\frac{C J C}{c j c}\right]^{2 / N C} \\
& \times \tau \text { nalC }-2 / N C \times \exp \left(p \times \frac{2}{N C} \times(T-\text { Tnom })\right)
\end{aligned}
$$

where

$$
\text { isc }=I_{0}(T), I S C=I_{0}(\text { Tnom })
$$


$c j c$ and $C J C$ are defined in equation (2.4.11).

$\tau$ nalC is the nomalized lifetime of the base-collector junction.

$N C$ is the emission coefficient of the base-collector junction.

\section{CHARGE-CONTROL MODEL}

The Early effect presented in the total base charge will be discussed in this section. As presented in the section 1, the total saturation current depends inversely on the total base majority-charge, it is [4]:

$$
I_{s}=\frac{q^{2} A_{E}^{2} n_{i}^{2} \widetilde{D}_{n}}{Q_{B T}}
$$

, and the total base charge in the active region is:

$$
Q_{B T}=Q_{B 0}+Q_{E}+Q_{C} \frac{A_{E}}{A_{C}}+Q_{F}+Q_{R}
$$

In addition to the built-in base charge, the total base charge presents the charge affected by both Early effect and high-current effect. Early effect is a consequence of the base-width variation with applied voltage under low-level injection/reverse bias conditions. $Q_{E}$ and $Q_{C}$ are emitter and collector charge-storage contributions.

After normalizing by dividing the built-in base charge, the total base charge becomes:

$$
\begin{aligned}
& q_{b}=\frac{Q_{B}}{Q_{B 0}}=1+\frac{Q_{E}}{Q_{B 0}}+\frac{Q_{C}}{Q_{B 0}} \frac{A_{E}}{A_{C}}+\frac{Q_{F}}{Q_{B 0}}+\frac{Q_{R}}{Q_{B 0}} \\
&=q_{1}+\frac{q_{2}}{q_{b}} \\
& q_{b} \cong \frac{q_{1}\left(1+\sqrt{1+4 q_{2}}\right)}{2}
\end{aligned}
$$

where

$$
q_{1}=1+\frac{Q_{E}}{Q_{B 0}}+\frac{Q_{C}}{Q_{B 0}} \frac{A_{E}}{A_{C}}
$$




$$
\frac{q_{2}}{q_{b}}=\frac{Q_{F}}{Q_{B 0}}+\frac{Q_{R}}{Q_{B 0}}
$$

To present the performance of the base-width modulations, equation (2.6.2) will be represented by a expression which is described below.

In equation (2.6.2) $Q_{E}$ and $Q_{C}$ are defined as follows,

$$
Q_{E}=\int_{0}^{V_{B E}} C_{j e}(V) d V \quad \text { and } \quad Q_{C}=\int_{0}^{V_{B C}} C_{j c}(V) d V
$$

, and $Q_{E}$ and $Q_{C}$ can also be represented by the simple, approximate fomulas, which are:

$$
\begin{aligned}
& Q_{E}=\int_{0}^{V_{B E}} C_{j e}(V) d V \cong \sum_{0}^{V_{B E}} C_{j e}(V) \Delta V=\left[C_{j e}\left(V_{B E}\right)-C_{j e}(0)\right] V_{B E} \\
& Q_{C}=\int_{0}^{V_{B C}} C_{j c}(V) d V \cong \sum_{0}^{V_{B C}} C_{j c}(V) \Delta V=\left[C_{j c}\left(V_{B C}\right)-C_{j c}(0)\right] V_{B C}
\end{aligned}
$$

Substitution of equations (2.6.5) and (2.6.6) into (2.6.2) gives

$$
\begin{aligned}
q_{1} & =1+\frac{V_{B E}}{\left|\frac{Q_{B 0}}{C_{j e}\left(V_{B E}\right)-C_{j e}(0)}\right|}+\frac{V_{B C}}{\left|\frac{Q_{B 0}}{C_{j c}\left(V_{B C}\right)-C_{j c}(0)}\right|\left[\frac{A_{C}}{A_{E}}\right]} \\
& =1+\frac{V_{B E}}{\left|\frac{Q_{B 0}}{\bar{C}_{j e}}\right|}+\frac{V_{B C}}{\left|\frac{Q_{B 0}}{\bar{C}_{j c}}\right| \times A C} \\
& =1+\frac{V_{B E}}{V A R}+\frac{V_{B C}}{V A F}
\end{aligned}
$$

where

$\bar{C}_{j e}$ and $\bar{C}_{j c}$ are average capacitances. 


$$
\begin{aligned}
& \bar{C}_{j e}=\frac{\int_{0}^{V_{B E}} C_{j e}(V) d V}{V_{B E}} \text { and } \bar{C}_{j c}=\frac{\int_{0}^{V_{B C}} C_{j c}(V) d V}{V_{B C}} \\
& A C=\frac{A_{C}}{A_{E}}
\end{aligned}
$$

$V A R$ is reverse Early voltage and $V A F$ is forward Early voltage.

Since the average capacitances are bias dependent, the values of Early voltages are not constants, as defined in the present model. Therefore, the expression of biasdependent Early effect is defined in the GP2 model as follows,

$$
o q_{1}=1-\frac{V_{B E}}{\left|\frac{Q_{B 0}}{\bar{C}_{j e}}\right|}-\frac{V_{B C}}{\left|\frac{Q_{B 0}}{\bar{C}_{j c}}\right| \times A C}
$$

where

$$
o q_{1}=\frac{1}{q_{1}}
$$

Up to this section, all the equations for the temperature-dependent parameters and the bias-dependent Early effect in the section of the charge-control model have been derived. Their applications will be discussed in the following section.

\section{APPLICATION}

The application of all of the parameters derived above will be discussed in this section. Some of the simplified, rather than complicated, equations will be applied. The input parameters and their default values will be decided as well. In each of the following sections, the parameter equation will be listed and its application discussed.

\section{Junction saturation current}

$$
I s(T)=I s(\text { Tnom }) \times(M T)^{3 / 2} \times(\text { ratio })^{4} \times\left(U T_{n}\right) \times \exp \left(\frac{E G B}{V_{t}}(\text { ratio }-1)\right)
$$


If nominal temperature is taken at $300 \mathrm{~K}$, the terms of effective mass and mobility, $M T$ and $U T_{n}$, can be represented by the simplified expressions, obtained from comparing the values of the equations (2.1.17), (2.1.18) and (2.1.20). These simplified expressions are:

$$
\begin{aligned}
& M T \cong\left(\frac{T}{300}\right)^{0.31} \\
& U T_{n} \cong\left(\frac{T}{300}\right)^{-0.57}\left(\frac{T}{300}\right)^{X T U_{n}}
\end{aligned}
$$

where

$X T U n$ is the temperature coefficient and varies with the dopant concentrations.

All the temperature coefficients can be represented by $X T I$. It is:

$X T I=4+0.465-0.57+X T U n$ for an npn transistor

or

$$
X T I=4+0.465-0.57+X T U p \quad \text { for a pnp transistor }
$$

Substitution of $X T I$ into equation (2.7.1) gives

$$
\text { is }=I S \times A R E A \times(\text { ratio })^{X T I} \times \exp \left(\frac{E G B}{V_{t}}(\text { ratio }-1)\right)
$$

where

Input parameters are $I S, A R E A, X T I$, and $E G B$.

$i s=I s(T), I S=I s(T n o m)$, and $A R E A$ is the emitter area factor.

The default value for $A R E A=1$ and for $E G B=1.176 \mathrm{eV}$. For an npn transistor, the default value of $X T I=4.065$, for a pnp transistor, $X T I=3.945$. (The average dopant concentrations in the base is assumed equal to $5 \times 10^{17} \mathrm{~cm}^{-3}$.)

\section{Ideal current gain}

Equation for the forward current gain is:

$$
\beta_{F}(T)=\beta_{F}(\text { Tnom }) \times\left(U T_{n}\right) \times\left(U T_{p}\right)^{-1} \times \exp \left(\frac{\Delta E G E}{V_{t}}(1-\text { ratio })\right)
$$


If the nominal temperature is taken at $300 \mathrm{~K}$, the terms of mobilities for electrons and holes can be represented by the simplified expressions. The expression for electron mobility has been defined in equation (2.7.3), the expression for hole mobility is shown as follows,

$$
U T_{p} \cong\left(\frac{T}{300}\right)^{-0.57}\left(\frac{T}{300}\right)^{X T U_{p}}
$$

where

$X T U p$ is the temperature coefficient and varies with the dopant concentrations.

Substitution equations (2.7.3) and (2.7.8) into (2.7.7) gives

$$
b f=B F \times(\text { ratio })^{X T B F} \times \exp \left(\frac{\Delta E G E}{V_{t}}(1-\text { ratio })\right)
$$

where

Input parameters are $B F, X T B F$, and $E G E$.

$b f=\beta_{F}(T), B F=\beta_{F}($ Tnom $), \Delta E G E=E G E-E G B$, and $X T B F=X T U n-$ $X T U p$ for an npn transistor or $X T B F=X T U p-X T U n$ for a pnp transistor.

The default value of $X T B F=0.04$ for an npn transistor and $X T B F=0.03$ for a pnp transistor. The default value of $E G E=1.081 \mathrm{eV}$.

$\triangle E G E=-0.095 \mathrm{eV}$. (The average dopant concentrations in the emitter is assumed equal to $10^{20} \mathrm{~cm}^{-3}$.)

Equation for the reverse current gain is:

$$
\beta_{R}(T)=\beta_{R}(\text { Tnom }) \times\left(U T_{n}\right) \times\left(U T_{p}\right)^{-1} \times \exp \mid \frac{\Delta E G C}{V_{t}}(1-\text { ratio }) \mid
$$

By applying simplified equations (2.7.3) and (2.7.8) to equation (2.7.10), the reverse current gain can be rewritten. It is:

$$
b r=B R \times(\text { ratio })^{X T B R} \times \exp \left(\frac{\Delta E G C}{V_{t}}(1-\text { ratio })\right)
$$

where 
Input parameter are $B R, X T B R$, and $E G C$.

$b r=\beta_{R}(T), B R=\beta_{R}($ Tnom $), \Delta E G C=E G C-E G B$, and $X T B R=X T U n$

- XTUp for an npn transistor or $X T B R=X T U p-X T U n$ for a pnp transistor.

The default value of $X T B R=1.64$ for an npn transistor and $X T B R=1.48$ for a pnp transistor. The default value of $E G C=1.206 \mathrm{eV}$.

$\triangle E G C=0.03 \mathrm{eV}$. (The average dopant concentrations in the collector is assumed equal to $10^{16} \mathrm{~cm}^{-3}$.)

Since different dopant concentrations have been assumed in the emitter, base, and collector, the variant values of mobilities in these three regions are obtained which results in the wide differences between $X T B F$ and $X T B R$.

\section{Built-in junction potential}

$$
\begin{aligned}
& v j e=V J E \times \text { ratio }- \text { vref } \\
& v j c=V J C \times \text { ratio }-v r e f \\
& v j s=V J S \times \text { ratio }- \text { vref }
\end{aligned}
$$

where

$$
\text { vref }=\frac{3}{2} V_{t} \times \log _{e}\left(M T \times(\text { ratio })^{2}\right)-E G(1-\text { ratio })
$$

If the nominal temperature is taken at $300 \mathrm{~K}, M T$ in equation (2.7.15) can be represented by simplified expression defined in equation (2.7.2). Substitution of equation (2.7.2) into $M T$ of (2.7.15) gives

$$
\text { vref }=\frac{3}{2} V_{t} \times \log _{e}\left((\text { ratio })^{X T V}\right)-E G(1-\text { ratio })
$$

where

Input parameters are $V J E, V J C, V J S, X T V$, and $E G$.

$X T V$ is temperature coefficient and related to the nominal temperature. 
The default values for $X T V=2.31$, for $E G=1.206 \mathrm{eV}$.

\section{Zero-bias junction capacitance}

$$
\begin{aligned}
& c j e=C J E \times \exp (p \times(1-M J E) \times(T-T n o m)) \times\left(\frac{v j e}{V I E}\right)^{-M J E} \\
& c j c=C J C \times \exp (p \times(1-M J C) \times(T-T n o m)) \times\left(\frac{v j c}{V I C}\right)^{-M J C} \\
& c j s=C J S \times \exp (p \times(1-M J S) \times(T-T n o m)) \times\left(\frac{v j S}{V I S}\right)^{-M J S}
\end{aligned}
$$

If the nominal temperature is taken at $300 \mathrm{~K}$, equations (2.7.12-16) are applied in equations (2.7.17-19). The exponential terms in equations (2.7.17-19) can also be simplified. Their applications are listed below. The equation for emitter-base junction is in (2.7.20), for base-collector junction in (2.7.21), and for collector-substrate junction in (2.7.22).

$$
\begin{aligned}
& c j e=C J E \times A R E A \times[1+p \times(1-M J E) \times(T-\text { Tnom })] \times\left(\frac{v j e}{V J E}\right)^{-M J E} \\
& c j c=C J C \times A R E A \times[1+p \times(1-M J C) \times(T-\text { Tnom })] \times\left(\frac{v j c}{V J C}\right)^{-M J C} \\
& c j s=C J S \times A R E A \times[1+p \times(1-M J S) \times(T-\text { Tnom })] \times\left(\frac{v j s}{V J S}\right)^{-M J S}
\end{aligned}
$$

where

Input parameters are $C J E, C J C, C J S, p, M J E, M J C$, and $M J S$.

The default values for $M J E=1 / 3, M J C=1 / 2, M J S=1 / 2$, and $p=7.8 \times 10^{-5}$.

\section{Leakage saturation current}

$$
\text { ise }=I S E \times M T^{3 / 2 N E} \times \text { ratio }^{3 / N E} \times \exp \left(\frac{E G}{N E V_{t}}(\text { ratio }-1)\right) \times\left[\frac{C J E}{c j e}\right]^{2 / N E}
$$




$$
\begin{gathered}
\times \tau \text { nale }-2 / N E \times \exp \left(p \times \frac{2}{N E} \times(T-\text { Tnom })\right) \\
\text { isc }=I S C \times M T^{3 / 2 N C} \times \text { ratio }^{3 / N C} \times \exp \left(\frac{E G}{N C V_{t}}(\text { ratio }-1)\right) \times\left[\frac{C J C}{c j C}\right]^{2 / N C} \\
\times \tau \text { nalC }^{-2 / N C} \times \exp \left(p \times \frac{2}{N C} \times(T-\text { Tnom })\right)
\end{gathered}
$$

If the nominal temperature is taken at $300 \mathrm{~K}, X T V$ in equation (2.7.16) and zerobias junction capacitance in equations (2.7.20-21) are applied in equations (2.7.23-24). The last exponential terms in equations (2.7.23-24) can also be simplified. Their applications are listed below. The equation for emitter-base junction is in (2.7.25) and for base-collector junction in (2.7.26).

$$
\begin{aligned}
& \text { ise }=I S E \times A R E A \times\left[\frac{C J E}{c j e}\right]^{2 / N E} \times \tau \text { nalE }-2 / N E \times \text { iseref } \\
& \text { isc }=I S C \times A R E A \times\left[\frac{C J C}{c j c}\right]^{2 / N C} \times \tau \text { nalC }-2 / N C \times \text { iscref }
\end{aligned}
$$

where

$$
\begin{aligned}
& \text { iseref }=(\text { ratio })^{3 X T V / 2 N E} \times\left[1+p \times \frac{2}{N E} \times(T-\text { Tnom })\right] \\
& \quad \times \exp \left(\frac{E G}{N E V_{t}}(\text { ratio }-1)\right) \\
& \text { iscref }=(\text { ratio })^{3 X T V / 2 N C \times\left[1+p \times \frac{2}{N C} \times(T-\text { Tnom })\right]} \\
& \quad \times \exp \left(\frac{E G}{N C V_{t}}(\text { ratio }-1)\right)
\end{aligned}
$$

Input parameters are ISE, ISC, $\tau$ nalE, $\tau$ nalC, $N E$, and $N C$.

The default values are: $N E=N C=2$.

\section{Charge-control model}

$$
o q_{1}=1-\frac{V_{B E}}{\left|\frac{Q_{B 0}}{\bar{C}_{j e}}\right|}-\frac{V_{B C}}{\left|\frac{Q_{B 0}}{\bar{C}_{j c}}\right| \times A C}
$$


where

$$
\begin{aligned}
& \bar{C}_{j e}=\frac{Q_{E}}{V_{B E}}=\frac{\int_{0}^{V_{B E}} C_{j e}(V) d V}{V_{B E}} \\
& \bar{C}_{j c}=\frac{Q_{C}}{V_{B C}}=\frac{\int_{0}^{V_{B C}} C_{j c}(V) d V}{V_{B C}}
\end{aligned}
$$

The integrations in equations (2.7.30-31) can be performed by the functional model inside the GP2 model and the average capacitances can be represented as follows,

$$
\begin{aligned}
& \bar{C}_{j e}=\frac{Q_{E}}{V_{B E}}=\frac{\text { SpiceDepletionCharge }\left(V_{B E}, \text { cje, vje, MJE, FC }\right)}{V_{B E}} \\
& \bar{C}_{j c}=\frac{Q_{C}}{V_{B C}}=\frac{\text { SpiceDepletionCharge }\left(V_{B C},\right. \text { cjc, vjc, MJC, FC) }}{V_{B C}}
\end{aligned}
$$

According to equations (2.7.29-33), the section of "calculate the normalized base charge" in the GP2 model will be rewritten as follows,

$$
\begin{aligned}
& Q E=\text { SpiceDepletionCharge }\left(V_{B E}, \text { cje, vje, MJE, } F C\right) \\
& Q C=\text { SpiceDepletionCharge }\left(V_{B C}, \text { cjc, vjc, MJC, FC }\right) \\
& \text { if }\left(V_{B E} \neq 0 \& V_{B C} \neq 0\right) \\
& \left\{\text { cjeval }=\frac{Q E}{V_{B E}} ; \text { cjcval }=\frac{Q C}{V_{B C}} ;\right. \\
& \left.o q_{1}=1-\frac{V_{B E}}{\left|\frac{Q_{B 0}}{\text { cjeval }}\right|}-\frac{V_{B C}}{\left|\frac{Q_{B 0}}{\text { cjcval }}\right| \times A C}\right\}
\end{aligned}
$$

else if $\left(V_{B E}=0 \& V_{B C} \neq 0\right)$ 
$\left\{\right.$ cjcval $\left.=\frac{Q C}{V_{B C}} ; o q_{1}=1-\frac{V_{B C}}{\left|\frac{Q_{B 0}}{\text { cjcval }}\right| \times A C}\right\}$

else if $\left(V_{B E} \neq 0 \& V_{B C}=0\right)$

$\left\{\right.$ cjeval $\left.=\frac{Q E}{V_{B E}} ; o q_{1}=1-\frac{V_{B E}}{\left|\frac{Q_{B 0}}{\text { cjeval }}\right|}\right\}$

else if $\left(V_{B E}=0 \& V_{B C}=0\right)$

$\left\{o q_{1}=1\right\}$

$a r g=o i k f \times i b e l+o i k r \times i b c l$

if $(\arg >0)$

$\left\{o q b=2 \times o q_{1} /(1+\operatorname{sqrt}(1+4 \times \arg ))\right\}$

else

$\left\{o q b=o q_{1}\right\}$

where

Input parameters are $Q_{B 0}$ and $A C$. 


\section{CHAPTER III}

\section{DISCUSSION AND COMPARISON}

In this chapter, the differences in equations between the present model and the model rederived in Chapter II will be investigated. The two models differ due to the way certain effects, such as effective mass, mobility, energy gap, bandgap narrowing, dielectric constant, and lifetime, are incorporated in these models. A comparison of these differences will be presented.

\section{JUNCTION SATURATION CURRENT}

The equation of the junction saturation current in the present model is identical to the one in the rederived model but the default values of input parameters are different.

\section{Present model}

$$
i s=I S \times A R E A \times \exp \left(\frac{E G \times(\text { ratio }-1)}{V_{t}}\right) \times \text { ratio }^{X T I}
$$

Input parameters are $I S, A R E A, E G$, and $X T I$.

The default values are: $E G=1.11 \mathrm{eV}, X T I=3$, and $A R E A=1$.

\section{Rederived model}

$$
\text { is }=I S \times A R E A \times \exp \left(\frac{E G B \times(\text { ratio }-1)}{V_{t}}\right) \times \text { ratio } X T I
$$

Input parameters are $I S, A R E A, E G B$, and $X T I$.

The default values are: $X T I=4.065$ for an npn transistor and $X T I=3.945$ for a pnp transistor. $E G B=1.176 \mathrm{eV}$, and $A R E A=1$.

The input parameters which have different default values for each model are 
temperature exponent $X T I$ and energy gap, $E G$ is for the present model and $E G B$ for the rederived model. To understand the differences in default values of input parameters between the two models, first of all, the definition of the junction saturation current is shown as follows,

$$
I s=\frac{q A_{E} n_{i}^{2} \widetilde{D}_{n}}{N_{A}^{B}}
$$

If both of effective mass and diffusion coefficient are assumed temperature independent and energy gap is used as in equation (3.1.7), equation (3.1.3) will be defined as follows,

$$
I s(T)=\frac{q A_{E} \widetilde{D}_{n}}{N_{A}^{B}} n_{i}^{2}(T)=\text { const } . \times T^{3} \times \exp \left(-\frac{E_{g}(T)}{V_{t}}\right)
$$

Using the same processes of derivation and integration in Chapter II, equation (3.1.4) becomes:

$$
i s=I S \times A R E A \times \exp \left(\frac{E G \times(\text { ratio }-1)}{V_{t}}\right) \times \text { ratio }^{3}
$$

The default value of $X T I$ in the present model was obtained from the temperature exponent, 3, in equation (3.1.5). The different default value of XTI was obtained for rederived model because the temperature behavior of effective mass and diffusion coefficient was included in XTI, defined in equation (2.7.4) or (2.7.5) for an npn or a pnp transistor. The default value of $E G$ in the present model is the experimental value of energy gap at $300 \mathrm{~K}$ [1]. Actually, $E G$ in equation (3.1.5) is the energy gap at $0 \mathrm{~K}$ not at $300 \mathrm{~K}$. For silicon, $E G$ is equal to $1.206 \mathrm{eV}$ if equation (3.1.7) for energy gap is used. $E G$ is defined as an input parameter so that it can be valid for other semiconductor materials. In the rederived model, in order to distinguish the energy gap without bandgap narrowing, used in the built-in junction potential, $E G B$ in equation (3.1.2) stands for the energy gap at $0 \mathrm{~K}$ with the bandgap narrowing and $E G B$ is defined in equations (2.1.6) and (2.1.7). Considering the default values of $X T I$ and $E G$ in the present model, it appears that the model was derived without including the temperature behavior of effective 
mass, diffusion coefficient, and bandgap narrowing. The default value of $E G$ is also questionable.

The more detailed temperature behavior of energy gap, bandgap narrowing, effective mass, and mobility will be discussed below.

\section{Energy gap}

Energy-gap equation used in the built-in junction potential, equation (3.3.5), for the present model was derived by Varshni [16]. The constants in this equation had been improved by Thurmond [17], using the thermodynamics relationships and the experimental data from Bludau et al [18] and Shaklee et al [19] to fit the Varshni's equation for silicon and germanium within the temperature range of 0 to $500 \mathrm{~K}$. Thurmond stated that the obtained value of constant $\beta$ for silicon is a more reasonable approximation to the Debye temperature, which determines the consistency between the Varshni's equation and the theoretical results. This general formula for energy gap is:

$$
E_{g}=E G-\frac{\alpha T^{2}}{T+\beta}
$$

where

For silicon, $E G=1.17 \mathrm{eV}, \alpha=4.73 \times 10^{-4}, \beta=636$, and $T=[0,500] \mathrm{K}$.

The expression for energy gap adopted in the rederived model has been used in Slotboom et al [7] to investigate experimentally the bandgap narrowing. They took the approximate expression for energy gap in the range of temperature from 250 to $500 \mathrm{~K}$ since energy gap as linear function of temperature can be procured when temperature reaches higher than $250 \mathrm{~K}$. This approximate equation is:

$$
E_{g}=E G-\alpha T
$$

where

$$
E G=1.206 \mathrm{ev} \text { and } \alpha=2.8 \times 10^{-4}[20]
$$

Approximated values for two energy gaps in the range of $[250,500] \mathrm{K}$ are shown 
in Figure 1.

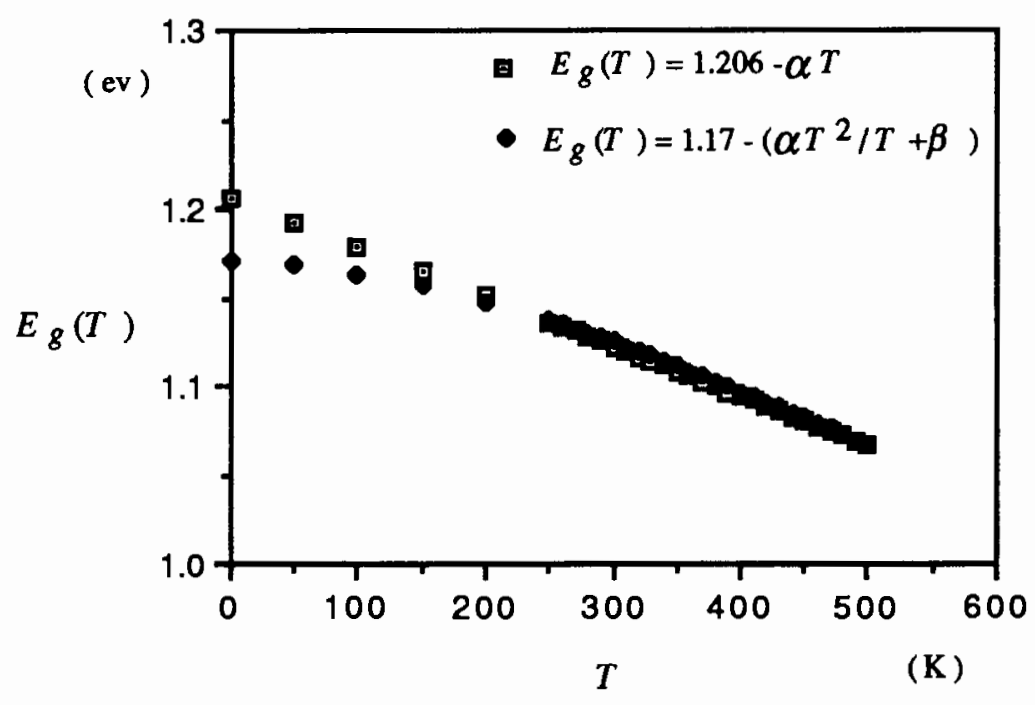

Figure 1. Energy gap as function of temperature for two different expressions.

If two different expressions of energy gaps, equation (3.1.6) and (3.1.7), are applied in the junction saturation current, the exponential terms for energy gaps can be obtained. The exponential term for approximate energy-gap equation, equation (3.1.7), is shown in equation (3.1.2), and the exponential terms for the general energy-gap equation, equation (3.1.6), are shown as follows,

$$
\text { is }=I S \times A R E A \times \text { ratio }^{X T I} \times \exp \left(\frac{E G \times(\text { ratio }-1)}{V_{t}}\right) \times \exp \left(\frac{\alpha \beta}{V_{t \beta}}\left(T_{\beta}-1\right)\right)
$$

where

$$
V_{t \beta}=\frac{K(T+\beta)}{q} \quad \text { and } \quad T_{\beta}=\frac{T+\beta}{\text { Tnom }+\beta}
$$

When the two exponential terms in (3.1.8) are compared with the one in (3.1.2) within the range of $[250,500] \mathrm{K}$, it is found that the calculated values of two exponential terms are almost the same with those of one single exponential term. This result is shown in Figure 2. 


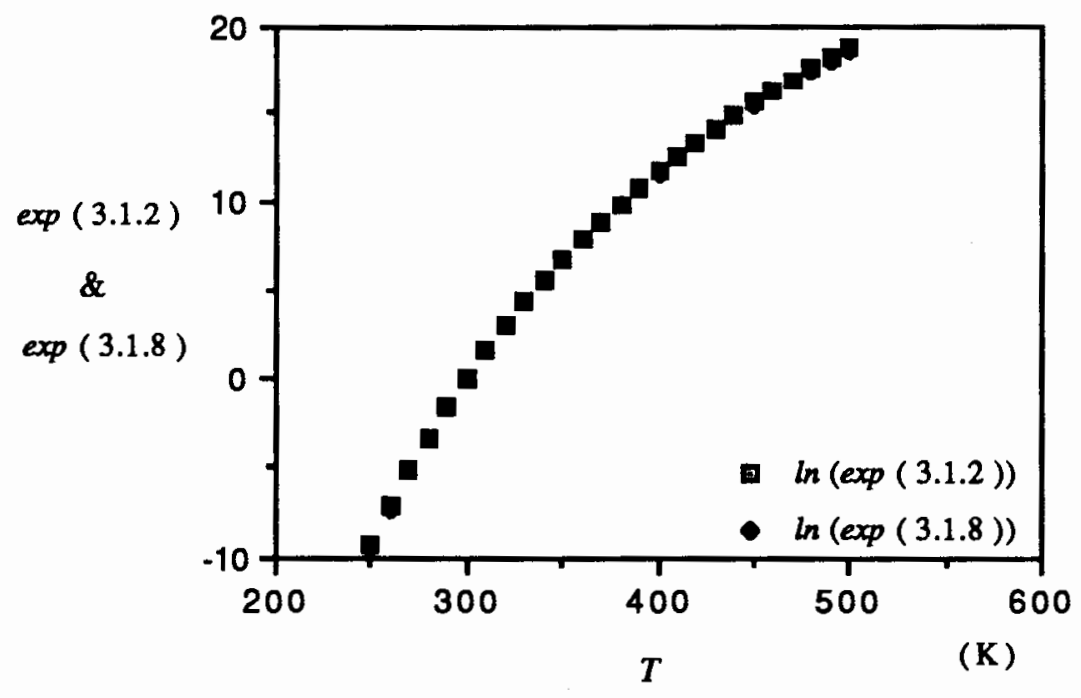

Figure 2. Comparison of exponential terms for energy gap.

Since the focus of this paper is on transistors operated under the temperature range of 250 to $500 \mathrm{~K}$, equation (3.1.2) instead of (3.1.8) is adopted within this range to simplify the equation of the junction saturation current and to reduce the input parameters from equations (3.1.8-9), such as $\alpha, \beta$. However, equation (3.1.8) can be applied to the much lower temperature range. Next, the bandgap narrowing will be discussed.

\section{Bandgap narrowing}

Bandgap narrowing is the prime shrinkage of the energy gap at high dopant concentrations. It is widely known that the concentrations of dopant impurities affect both the density of states associated with the host lattice and the density of states associated with the impurity atoms [21]. In the highly-doped silicon the energy-band structure changes due to the many-body effects that result in the broadening of the impurity band and the band-tail effects that result from the randomness of the impurity distribution on the edges of the conduction and valence bands [22]. These effects impact the energy gap in the emitter and the base region. An increase in the emitter doping level results in lower emitter efficiency and in increasing temperature dependence of the current gain [23].

Many papers have been written on the topic of bandgap narrowing. In addition to 
theory, three, mainly empirical, models investigating bandgap narrowing have been presented [24]. Three measurements are optical or infrared absorption measurements, electrical measurements, and photoluminescence measurement. Since different measurement methods have been used, there are a number of discrepancies in the quantitative data. The empirical formula, derived by Slotboom et al [7], is used in this paper since this formula was obtained by the characteristic of current-voltage from the electrical measurement. Also Wieder [25] found that it was satisfactory to use this formula to fit the experimental results with the exception of using a different value for constant $N_{0}$, because n-type material was used. After a more valid assumption, described by Fermi-Dirac statistics, was made for the density of the majority-carrier band in the high doping level, Mertens et al [26] indicated that the bandgap narrowing is not only a function of impurity concentration but also temperature dependent, and the temperaturedependence formula is added to the previous bandgap narrowing. Neugroschel et al [20] also found that the bandgap narrowing is temperature dependent when the Fermi-Dirac statistics are employed. However, Possin et al [27] used the experimental data within the temperature range from 212 to $371 \mathrm{~K}$ and suggested that the temperature-related part of bandgap narrowing, the Fermi-Dirac part, is not strongly temperature dependent and can be neglected. Thus this second-order part is not applied to this paper.

The formula of bandgap narrowing is shown as follows,

$$
\Delta E_{g}=9\left[\log _{e}\left(\frac{N}{N_{0}}\right)+\sqrt{\left[\log _{e}\left(\frac{N}{N_{0}}\right)\right]^{2}+0.5}\right](\mathrm{meV})
$$

where

$$
N_{0}=1 \times 10^{17} \text { for p-type and } N_{0}=1.5 \times 10^{17} \text { for n-type material semiconductors }
$$

Figure 3 presents the discrepancies of the bandgap-narrowing data, which were obtained from electrical measurements of Slotboom et al [7], Wieder [25], Mertens et al [26], and Neugroschel et al [20], from the luminescence measurement of Dumke [28], and from the theory of Fossum et al [29] who implied that the bandgap narrowing occurred above dopant concentrations equal to $10^{19} \mathrm{~cm}^{-3}$. 


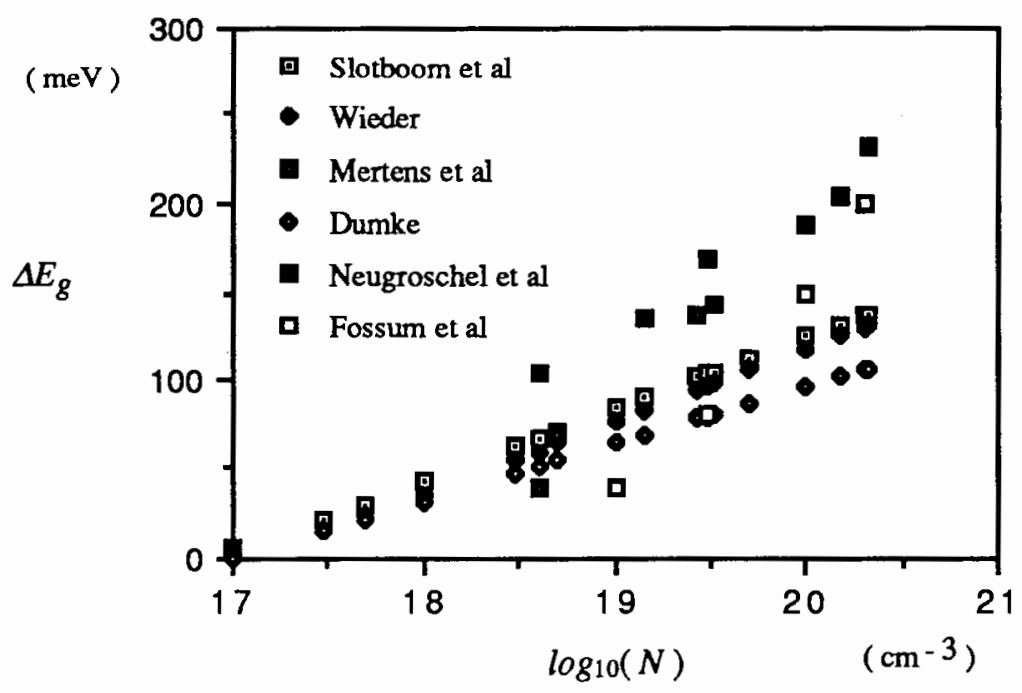

Figure 3. Summary of bandgap narrowing from different measurements. $N=\left[10^{17}, 2.1 \times 10^{20}\right] \mathrm{cm}^{-3}$

In the next section, the effective mass for electrons and holes dependence of temperature will be discussed.

\section{Effective mass}

Barber [5] reviewed the experimental data and theoretical expressions on the effective masses of electrons and holes for silicon. He concluded that both effective electron mass and hole mass are temperature and dopant concentration dependent. Expressions as a function of temperature for effective electron mass and hole mass were obtained by Gaensslen et al [6], referring the fitting polynomials to the Barber's data. These expressions represent the effective masses of the intrinsic carrier concentration. They are:

$$
\begin{aligned}
& m_{c}(T)=1.045+4.5 \times 10^{-4} T \\
& m_{v}(T)=0.523+1.4 \times 10^{-3} T-1.48 \times 10^{-6} T^{2}
\end{aligned}
$$

where

Equation (3.1.11) was obtained by fitting the experimental data, which show that 
the same temperature behavior of effective mass for electrons was obtained at the dopant concentrations less than $5 \times 10^{17} \mathrm{~cm}^{-3}$.

Equation (3.1.12) was obtained by fitting the theoretical calculations. The constant in the third term has been modified and is equal to $1.4 \times 10^{-6}$, used in equation

(2.1.4), in order to have the temperature behavior for equation (2.1.4) be valid over the temperature range from 50 to $500 \mathrm{~K}$.

When equations (3.1.11) and (3.1.12) are applied in the junction saturation current, they can be represented by a expression $M T$, equation (2.1.16). $M T$ is a complex equation. In order to easily model the junction saturation current and present the effect of temperature dependence of mass, $M T$ can be represented by a simplified equation, which is the ratio of the actual temperature to the nominal temperature. If the nominal temperature is taken at $300 \mathrm{~K}$, the values of the simplified equation are approximately the same with those of $M T$ within the range of 250 to $500 \mathrm{~K}$. Thus the simplified equation instead of $M T$ can be replaced in equation (2.1.15). Any temperature can be the nominal temperature. Since most of the experiments have been done at or near $300 \mathrm{~K}$ and usually transistors are operated in the circuit at the room temperature (about $300 \mathrm{~K}$ ), typically the nominal temperature is taken at $300 \mathrm{~K}$. For instance, the simplified equation will be: $(T / 350)^{0.35}$, if the nominal temperature is taken at $350 \mathrm{~K}$. If the nominal temperature is taken at $300 \mathrm{~K}$, the simplified equation is shown in equation (2.7.2) and its temperature coefficient equals 0.31 , which is: $(T / 300)^{0.31}$. In the present model, the nominal temperature is taken at $300 \mathrm{~K}$ and this number is fixed in the program. To correct this shortcomings, an input parameter for the nominal temperature is added in the program.

The comparison of $M T$ with the simplified equation, equation (2.7.2), is shown in Figure 4.

Next, the temperature-dependent mobility will be discussed. 


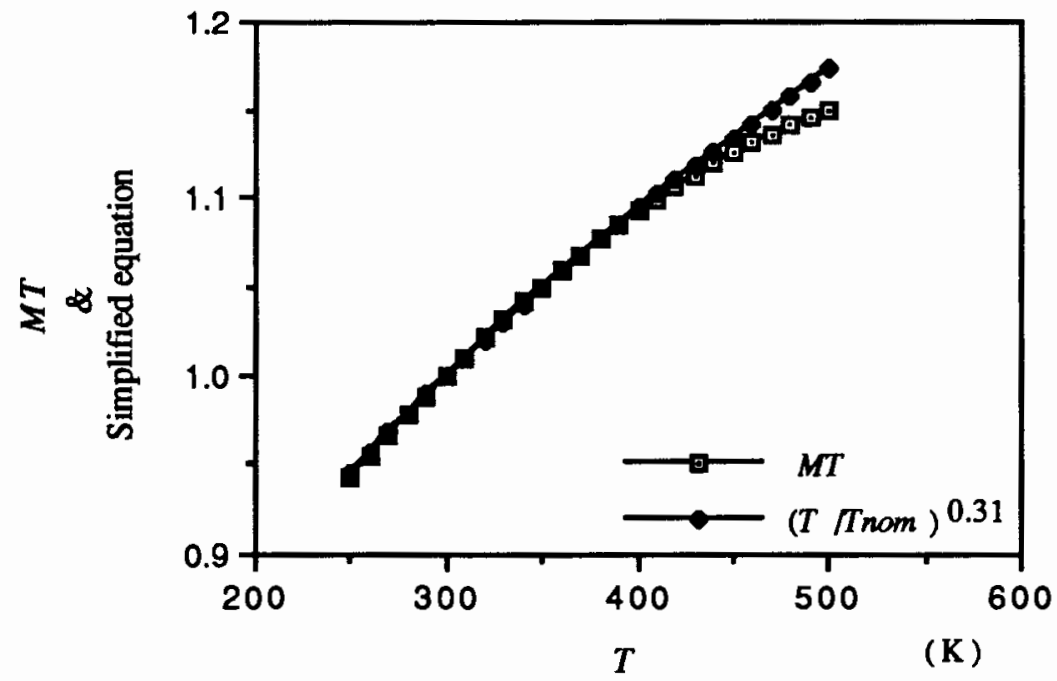

Figure 4. Comparison of the simplified equation with effective-mass ratio.

\section{Mobility}

The average diffusion coefficient can be expressed as a function of mobility through the Einstein relation, equation (2.1.8). This mobility in the formula of the junction saturation current represents the mobility of minority carriers in the base. For an npn transistor the minority carriers are electrons diffusing in the p-type base, and for a pnp transistor the minority carriers are holes diffusing in the n-type base. Since there is no expression describing temperature behavior for minority-carrier mobilities, the expressions of majority-carrier mobilities representing those of minority-carrier mobilities have been used in the junction saturation current. These expressions were derived by Arora et al [8] from both experimental data and theory of mobility. These expressions are a function of impurity concentration and temperature. They are shown in equations (2.1.9) and (2.2.3) and are valid over the temperature range of 250 to $500 \mathrm{~K}$. The differences in mobility between minority and majority carriers are addressed in the following section.

Many articles relate to carrier mobility but most of them concentrate on the mobility as a function of the dopant concentrations. For electrons, the comparable values for 
minority- and majority-carrier mobilities were experimentally found by Dziewior et al [30], Burk et al [31], and Neugroschel [32] within the doping range of $10^{14}$ to $3.5 \times$ $10^{18} \mathrm{~cm}^{-3}$ at $300 \mathrm{~K}$. Within high doping range, $10^{19}$ to $10^{20} \mathrm{~cm}^{-3}$, Swirhun et al [33] indicated that the values of minority-carrier mobility are two and half times larger than those of the corresponding majority-carrier mobility. For holes, they [30, 31, 32] found that the minority-carrier mobilities are larger than the majority-carrier mobilities within the doping range of $10^{14}$ to $10^{19} \mathrm{~cm}^{-3}$. Within high doping range, $2 \times 10^{19}$ to $1.5 \times 10^{20}$

$\mathrm{cm}^{-3}$, Burk et al [31] and Neugroschel et al [34] discovered that the minority-carrier mobilities are less than the majority-carrier mobilities. These results were supported theoretically by Fossum et al $[29,35]$, who demonstrated that the minority-carrier mobilities in highly-doped material are temperature dependent. An opposite result to the Burk et al [31] and Neugroschel et al [34] was found by Del Alamo et al [36], indicating that the minority-carrier mobilities are two times larger than the majority-carrier mobilities within the high doping range, $10^{19}$ to $10^{20} \mathrm{~cm}^{-3}$.

Although different values for minority- and majority-carrier mobilities were obtained for a given dopant concentration, Burk et al [31], Neugroschel [32], and Swirhun et al [33] stated that the temperature behavior of minority-carrier mobility is similar to that of majority-carrier mobility. Therefore, the formulas of majority-carrier mobilities are acceptable to describe the temperature behavior of minority-carrier mobilities in the model equations. It is beyond the framework of this paper to obtain the empirical formulas, valid in the range of $[250,500] \mathrm{K}$, as a function of impurity concentration and temperature for the minority-carrier mobilities.

Complicated equations for mobilities of electrons and holes shown in (2.1.9), (2.1.20), (2.2.3), and (2.2.10) also can be simplified on the basis of the nominal temperature, for instance, at $300 \mathrm{~K}$. The simplified equations are obtained in equations (2.7.3) and (2.7.8), which are: $(T / 300)^{-0.57+X T U n}$ and $(T / 300)^{-0.57+X T U_{p}}$ where 
$X T U n$ and $X T U p$ are dopant-concentration-dependent coefficients. With a certain dopant concentration, approximately the same values can be obtained between the simplified equation and the equation of mobility within the range of 250 to $500 \mathrm{~K}$. Different dopant concentrations have different values for the temperature coefficient, XTUn or XTUp. If dopant concentration is taken at $10^{16} \mathrm{~cm}^{-3}, X T U n$ will equal -1.43 and XTUp equal

- 1.47. If dopant concentration is taken at $5 \times 10^{17} \mathrm{~cm}^{-3}, X T U n$ will equal 0.17 and XTUp 0.05 .

The comparisons of $U T_{n}$ and $U T_{p}$ with the simplified equations are shown in Figures 5 and 6 respectively. The dopant concentration is taken at $5 \times 10^{17} \mathrm{~cm}^{-3}$.

In the rederived model, supplied by the simplified equations of effective mass and mobility, the total temperature coefficient $X T I$ can present temperature behavior of effective mass and mobility. Since different temperature behaviors exist between holecarrier and electron-carrier mobilities, different values of XTI can be obtained either for an npn or a pnp transistor.

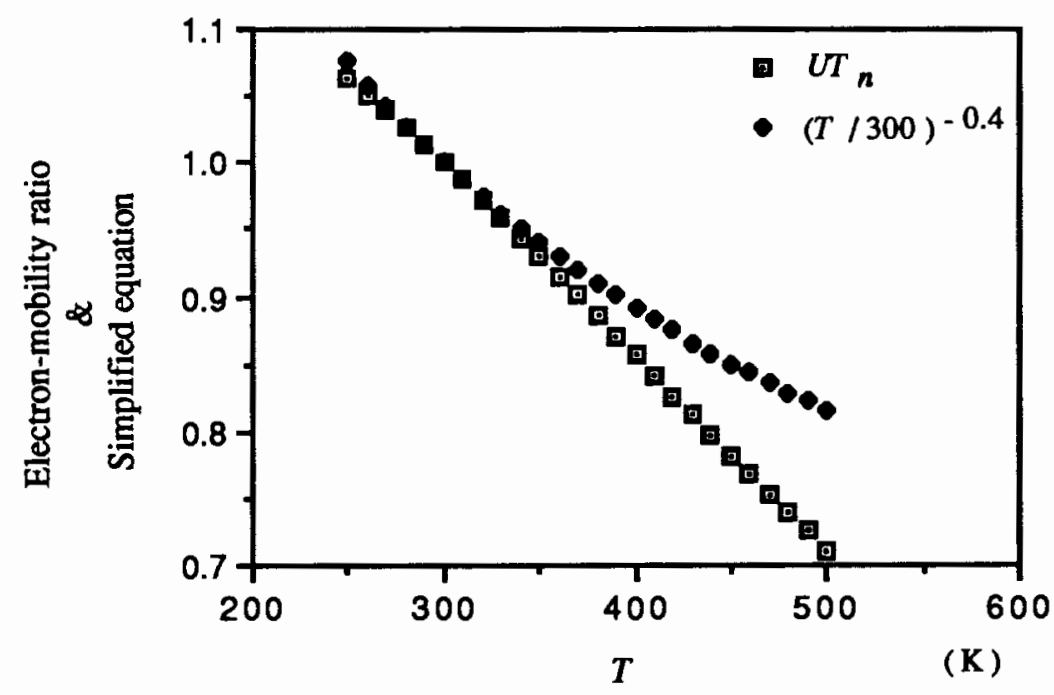

Figure 5. Comparison of the simplified equation with electron-mobility ratio. 


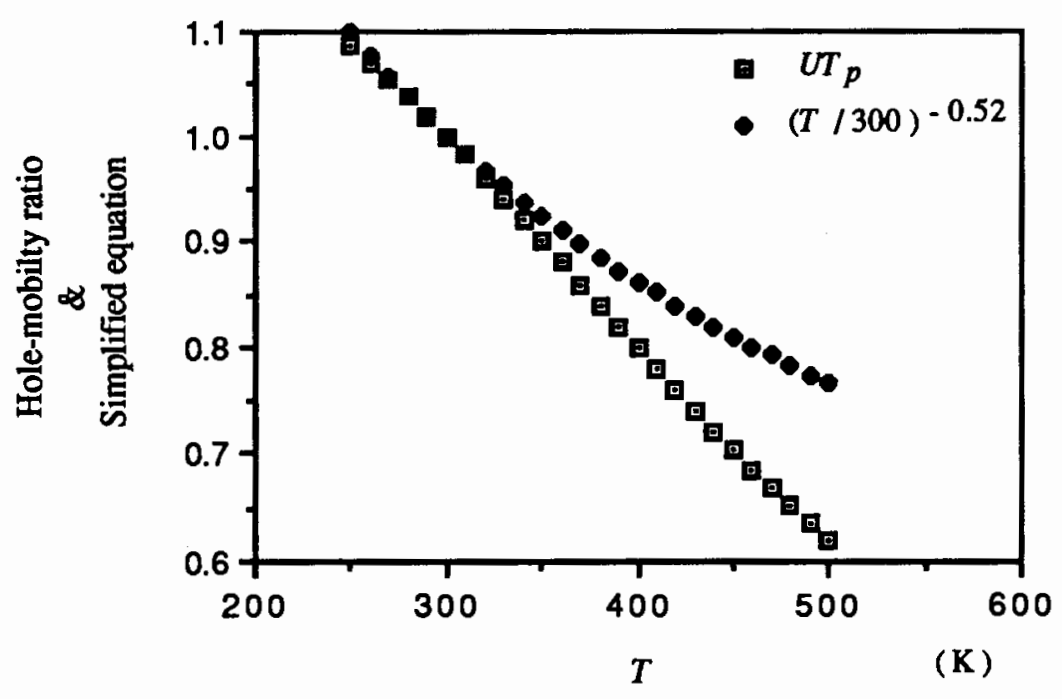

Figure 6. Comparison of the simplified equation with hole-mobility ratio.

\section{IDEAL CURRENT GAIN}

Present model

$b f=B F \times$ ratio $^{X T B}$ and $b r=B R \times$ ratio $^{X T B}$

Input parameters are $B F, B R$, and $X T B$.

The default value of $X T B$ is zero.

$\underline{\text { Rederived model }}$

$$
\begin{aligned}
& b f=B F \times \text { ratio }^{X T B F} \times \exp \left(\frac{\Delta E G E}{V_{t}}(1-\text { ratio })\right) \\
& b r=B R \times \text { ratio }^{X T B R} \times \exp \left(\frac{\Delta E G C}{V_{t}}(1-\text { ratio })\right)
\end{aligned}
$$

Input parameters are $B F, B R, X T B F, X T B R, E G E$, and $E G C$.

The default values are: $X T B F=0.04, X T B R=1.64$ for an npn transistor and $X T B F=0.03, X T B R=1.48$ for a pnp transistor.

The default values are: $E G E=1.081 \mathrm{eV}$ and $E G C=1.206 \mathrm{eV}$. 
The equations of the current gains in (3.2.1) for the present model are based on the empirical formula used by Idleman et al [37]. This formula is:

$$
\beta_{F}(T)=\beta_{F}(\text { Tnom })\left[1+T_{c 1} \Delta T+T_{c 2} \Delta T^{2}\right]
$$

where

$T_{c 1}$ and $T_{c 2}$ are empirical values. Typically, $T_{c 1}=0.0067 /{ }^{\circ} \mathrm{c}$ and

$$
T_{c 2}=0.000036 /\left({ }^{\circ} \mathrm{c}\right)^{2} . \Delta T=T-\text { Tnom } .
$$

A proper value will be obtained for $X T B$ after the comparison of equation (3.2.1) with (3.2.4) is made within the range of 250 to $500 \mathrm{~K}$. The value of $X T B$ is 1.85 .

One disadvantage for the present model is that the same parameter XTB is used in equations of the current gains, forward and reverse, i.e., the forward and reverse current gains can not exhibit a different temperature behavior. Since dopant concentrations in the emitter and collector are different, resulting in different temperature behavior for intrinsic carrier concentrations (bandgap narrowing) and mobilities, the forward/reverse current gains will exhibit a different temperature behavior.

The ideal current gain has been defined in Chapter II. It is the current gain where transistors operate at intermediate current levels. The temperature behavior of mobility associated with doping concentration and the temperature behavior caused by bandgap narrowing adequately explain the variances of the current gain with different temperature and dopant concentrations, especially when the current gain drops greatly at low temperature [38]. Therefore, equations in (3.2.2-3), rather than equation (3.2.1), are used to describe the temperature behavior of the current gains in the rederived model.

$X T B F$ and $X T B R$ are dopant-concentration dependent. For an npn transistor, for instant, $X T U n=0.17$ when $N a=5 \times 10^{17} \mathrm{~cm}^{-3}$ in the base, $X T U p=0.13$ when $N d=$ $10^{20} \mathrm{~cm}^{-3}$ in the emitter, and $X T U p=-1.47$ when $N d=10^{16} \mathrm{~cm}^{-3}$ in the collector. By the definitions of $X T B F$ for the forward current gain and $X T B R$ for the reverse current 
gain, $X T B F=X T U n-X T U p=0.17-0.13=0.04$ and $X T B R=X T U n-X T U p=0.17+$ $1.47=1.64$. Different dopant concentrations result in different temperature behavior of mobilities and wide difference in values between the current-gain coefficients, XTBF and $X T B R$. This wide difference creates a major distinguish in temperature behavior between the forward and reverse current gains.

\section{BUILT-IN JUNCTION POTENTIAL}

With the exception of the reference potential, the equations of the built-in junction potential for the present and rederived model are the same. The equations are shown below.

Emitter-base junction

$$
v j e=V J E \times \text { ratio }-v r e f
$$

Base-collector junction

$$
v j c=V J C \times \text { ratio }- \text { vref }
$$

Collector-substrate junction

$$
v j s=V J S \times \text { ratio }-v r e f
$$

\section{Present model}

$$
\begin{aligned}
& \text { vref }=3 \times V_{t} \times \log _{e}(\text { ratio })-e g+1.1150877 \times \text { ratio } \\
& e g=1.16-\frac{0.000702 \times T^{2}}{T+1108}
\end{aligned}
$$

Input parameters are VJE and VJC. 


\section{$\underline{\text { Rederived model }}$}

$$
\text { vref }=\frac{3}{2} V_{t} \times \log _{e}\left((\text { ratio })^{X T V}\right)-E G(1-\text { ratio })
$$

Input parameters are $V J E, V J C, V J S, X T V$, and $E G$.

The default values are: $X T V=2.31$ and $E G=1.206 \mathrm{eV}$.

The drawbacks in the present model and the differences between two models will be discussed.

The energy gap used in equation (3.3.4) is shown in (3.3.5). All constants in equation (3.3.5) are hardware numbers. In the last term of equation of reference potential, (3.3.4), the constant, 1.1150877 , is the value of the energy gap at $300 \mathrm{~K}$. All these numbers are only valid for silicon. If other semiconductor materials are used, because of these unchangeable numbers, the right values of the built-in junction potential can not be obtained. The way to correct these shortcomings is to change these fixed numbers to variables. Thus, equations (3.3.4) and (3.3.5) can be rewritten as follows,

$$
\begin{aligned}
& \text { vref }=3 \times V_{t} \times \log _{e}(\text { ratio })-e g+E_{g}(\text { Tnom }) \times \text { ratio } \\
& e g=E G-\frac{\alpha \times T^{2}}{T+\beta}
\end{aligned}
$$

where

Input parameters are: $E G, \alpha, \beta$, and $E_{g}($ Tnom).

The default values are: $E G=1.17 \mathrm{eV}, \alpha=0.000473, \beta=636$, and $E_{g}($ Tnom $)=E_{g}(300)=1.1245192$.

In order to avoid adding more input parameters into the program, the expression of energy gap as linear with temperature above $250 \mathrm{~K}$, equation (3.1.7), is used in the rederived model. This is why a difference exists in the parameters for energy gap in the equation of reference potential between the two models. Since temperature dependence of effective mass has been taken into account in the reference potential for the rederived 
model, the other difference in the equation of reference potential between the two models is that there is an input parameter $X T V$ in the rederived model but not in the present model. The built-in junction potential for the collector-substrate junction has been introduced in the rederived model but not in the present model. It exists in the active region between the junction of collector and substrate for an npn transistor so that the capacitance of collector-substrate can be described.

\section{ZERO-BIAS JUNCTION CAPACITANCE}

The equations of the zero-bias junction capacitance in the present model were obtained under three assumptions, which will be discussed. The zero-bias junction capacitance are represented in three junctions, emitter-base, base-collector, and collectorsubstrate. First of all, they will be listed as follows.

\section{Present model}

$$
\begin{aligned}
& c j e=C J E \times A R E A \times\left\{1+M J E \times\left[0.0004 \times(T-\text { Tnom })+\left(1-\frac{v j e}{V I E}\right)\right]\right\} \\
& c j c=C J C \times A R E A \times\left\{1+M J C \times\left[0.0004 \times(T-\text { Tnom })+\left(1-\frac{v j c}{V J C}\right)\right]\right\} \\
& c j s=C J S \times A R E A
\end{aligned}
$$

Input parameters are $C J E, C J C, C J S, M J E$, and $M J C$.

The default values are: $M J E=0.33$ and $M J C=0.33$.

\section{Rederived model}

$$
\begin{aligned}
& c j e=C J E \times A R E A \times[1+p \times(1-M J E) \times(T-\text { Tnom })] \times\left(\frac{v j e}{V J E}\right)^{-M J E} \\
& c j c=C J C \times A E R A \times\left[1+p \times(1-M J C) \times\left(T-T_{\text {nom }}\right)\right] \times\left(\frac{v j c}{V J C}\right)^{-M J C}
\end{aligned}
$$


$c j s=C J S \times A R E A \times[1+p \times(1-M J S) \times(T-T n o m)] \times\left(\frac{v j S}{V J S}\right)^{-M J S}$

Input parameters are $C J E, C J C, C J S, p, M J E, M J C$, and $M J S$.

The default values are: $M J E=0.33, M J C=0.5, M J S=0.5$, and $p=7.8 \times 10^{-5}$.

Since three assumptions have been made during the process of the derivation and the value of $\gamma_{T}^{\varepsilon}$ was fixed for silicon, different equations of the zero-bias junction

capacitance for the present model from those for the rederived model were obtained. The typical value of $\gamma_{T}^{\varepsilon}$ for silicon is $0.0002 /{ }^{\circ} \mathrm{c}$ [37]. Three assumptions are:

1. $\gamma_{T}^{\Phi_{i}}$ is assumed constant, i.e., $\gamma_{T}^{\Phi} \Phi_{i}=\gamma_{T \text { nom }}^{\Phi_{i}}$

2. A certain value is taken and incomplete substitution is made for the junction grading coefficient.

3. $e g$ is assumed equal to $E_{g}($ Tnom).

where

$$
\begin{aligned}
& \gamma_{T}^{\Phi_{i}}=\frac{1}{T}-\frac{K T}{q \Phi_{i}(T)}\left(\frac{3}{T}+\frac{E_{g}(T)}{K T^{2}}\right) \\
& \gamma_{\text {Tnom }}^{\Phi_{i}}=\frac{1}{\text { Tnom }}-\frac{K T_{\text {nom }}}{q \Phi_{i}(\text { Tnom })}\left(\frac{3}{\text { Tnom }}+\frac{E_{g}(\text { Tnom })}{K \text { Tnom }^{2}}\right)
\end{aligned}
$$

The zero-bias junction capacitance has been defined in equation (2.4.1) which is:

$$
C_{j 0}(T)=\frac{A \varepsilon^{1-m}(T)}{K_{c}^{\prime} \Phi_{i}^{m}(T)}
$$

Taking the derivative with respect to temperature of equation (3.4.9) gives

$$
\frac{\partial C_{j 0}(T)}{\partial T}=C_{j 0}(T) \times\left[\frac{1-m}{\varepsilon(T)} \frac{\partial \varepsilon(T)}{\partial}-\frac{m}{\Phi_{i}(T)} \frac{\partial \Phi_{i}(T)}{\partial T}\right]
$$




$$
\begin{array}{r}
=C_{j 0}(T) \times\left[(1-m) \times \gamma_{T}^{\varepsilon}-m \times \gamma_{T}^{\Phi_{i}}\right] \\
\frac{1}{C_{j 0}(T)}\left[\frac{\partial C_{j 0}(T)}{\partial T}\right]=(1-m) \times \gamma_{T}^{\varepsilon}-m \times \gamma_{T}^{\Phi_{i}}
\end{array}
$$

then

$$
\gamma_{T}^{C_{j 0}}=(1-m) \times \gamma_{T}^{\varepsilon}-m \times \gamma_{T}^{\Phi_{i}}
$$

Since the first-order temperature sensitivity of the built-in junction potential, $\gamma_{T}^{\Phi_{i}}$, is small, $\gamma_{T}^{\Phi_{i}}$ can be assumed equal to a constant, $\gamma_{\text {Tnom }}^{\Phi_{i}}[1], \gamma_{T}^{\varepsilon}$ varies constantly per Celsius degree [37]. Both of $\gamma_{T}^{\varepsilon}$ and $\gamma_{T \text { nom }}^{\Phi_{i}}$ are constant, thus, $\gamma_{T}^{C_{j 0}}$ of equation (3.4.10) is constant. If the values of this constant $\gamma_{T}^{C_{j 0}}$ are of little difference comparing with those of $\gamma_{T}^{C_{j 0}}$ varied with temperature from 250 to $500 \mathrm{~K}$, the assumption of constant for $\gamma_{T}^{\Phi_{i}}$ would be acceptable. From the comparison of the constant $\gamma_{T}^{C_{j 0}}$ with the varied $\gamma_{T}^{C_{j 0}}$ shown in figure 7, this assumption is questionable. The first assumption has been discussed above. The rest of the assumptions will be described in the end of this section. In the following the differences between two models will be discussed.

It is apparent that the effect of temperature dependence of effective mass is not included in the present model for the zero-bias junction capacitance. The constant $p$, related to the dielectric constant, in the rederived model is one of the input parameters so that, not only silicon, other semiconductor materials, such as germanium, can be applied in the program. The expression for dielectric constant is shown in equation (2.4.2). The temperature behavior of junction capacitance in the collector-substrate junction is the same with that in other junctions and the equation of the collector-substrate junction capacitance has been introduced in the rederived model but not in the present model. 


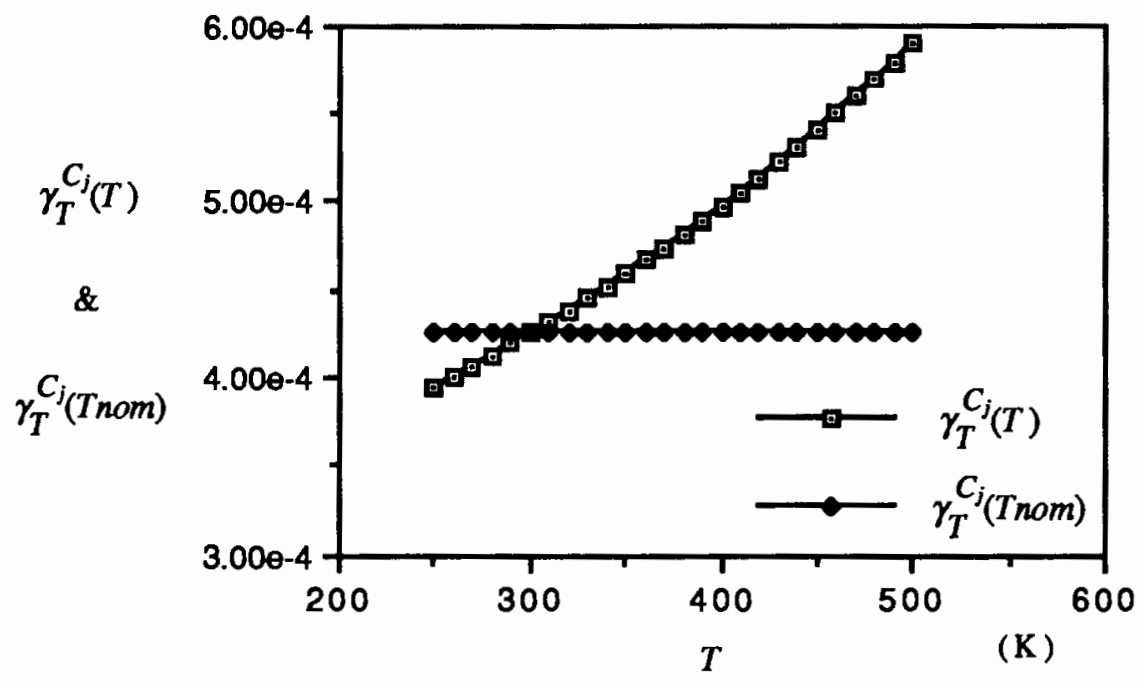

Figure 7. Comparison of the constant $\gamma_{T}^{C_{j 0}}$ with the varied $\gamma_{T}^{C_{j 0}}$ for the emitterbase junction.

If the effect of the contribution of heavily-doped-side carriers to the space charge in the lightly-doped region is considered, the offset voltage will be included in the zero-bias junction capacitance. The classical value $\Phi_{i}-2 V_{t}$ of the offset voltage for nearly

symmetrical junctions has been obtained by Gummel et al [39], investigating the offset voltage as a function of doping ratio and applied voltage for silicon at $300 \mathrm{~K}$. If the offset voltage is applied in the zero-bias junction capacitance, the rederived equation of the zerobias junction capacitance will be:

$$
\begin{aligned}
C_{j 0}(T)=C_{j 0}(\text { Tnom }) \times A R E A \times[1+p \times(1-m) \times(T-\text { Tnom })] \\
\times\left[\text { ratio }-\frac{v r e f}{\Phi_{i}(\text { Tnom })+2 \times \frac{K T \text { Tnom }}{q}}\right]^{-m}
\end{aligned}
$$

Since no offset voltage was found at other temperatures, the offset voltage was not applied in the rederived model.

The remaining assumptions will be presented below.

The first assumption has been made in equation (3.4.10). Calculating the definite 
integral with $C_{j}(T)$ and $C_{j}($ Tnom $)$ as limits gives

$$
\begin{aligned}
& \int_{C_{j}(\text { Tnom })}^{C_{j}(T)} \frac{d C_{j 0}(T)}{C_{j 0}(T)}=\int_{\text {Tnom }}^{T}\left[(1-m) \times \gamma_{T}^{\varepsilon}-m \times \gamma_{T}^{\Phi_{i}}\right] d T \\
& \ln \left(\frac{C_{j}(T)}{C_{j}(\text { Tnom })}\right)=\left[(1-m) \times \gamma_{T}^{\varepsilon}-m \times \gamma_{T}^{\Phi_{i}}\right](T-\text { Tnom }) \\
& \frac{C_{j}(T)}{C_{j}(\text { Tnom })}=\exp \left(\left[(1-m) \times \gamma_{T}^{\varepsilon}-m \times \gamma_{T}^{\Phi_{i}}\right](T-T \text { nom })\right) \\
& \cong 1+\left[(1-m) \times \gamma_{T}^{\mathcal{E}}-m \times \gamma_{T}^{\Phi_{i}}\right](T-\text { Tnom })+\ldots \\
& \frac{C_{j}(T)}{C_{j}(\text { Tnom })}=1+(T-\text { Tnom }) m\left[\left(\frac{1}{m}-1\right) \times \gamma_{T}^{\mathcal{E}}-\gamma_{T}^{\Phi_{i}}\right]
\end{aligned}
$$

In equation (3.4.12) $m$ is the junction grading coefficient. If the double-diffused transistor is applied, the junction is linearly graded so that $m$ is equal to $\frac{1}{3}$.

Substitution of $m=\frac{1}{3}$ into the term $\left(\frac{1}{m}-1\right)$ of equation (3.4.12) gives

$$
\frac{C_{j}(T)}{C_{j}(\text { Tnom })}=1+(T-\text { Tnom }) m\left(2 \gamma_{T}^{\varepsilon}-\gamma_{T}^{\Phi_{i}}\right)
$$

Equation (3.4.13) is exactly the same as formula (2.74) displayed by Getreu [1]. This is the second assumption: A certain value $m=\frac{1}{3}$ is taken and only $m$ of the term $\left(\frac{1}{m}-1\right)$ is substituted by $\frac{1}{3}$.

Equation (3.4.13) can be reorganized as follows,

$$
\begin{aligned}
\frac{C_{j}(T)}{C_{j}(\text { Tnom })} & =1+(T-\text { Tnom }) m\left(2 \gamma_{T}^{\mathcal{E}}-\gamma_{T}^{\Phi_{i}}\right) \\
& =1+m\left[2 \gamma_{T}^{\varepsilon}(T-\text { Tnom })-\gamma_{T}^{\Phi_{i}}(T-\text { Tnom })\right]
\end{aligned}
$$

where 


$$
\begin{aligned}
& \gamma_{T}^{\Phi_{i}}(T-\text { Tnom })=(T-\text { Tnom }) \times \\
& {\left[\frac{1}{\text { Tnom }}-\frac{K \text { Tnom }}{q \Phi_{i}(\text { Tnom })}\left(\frac{3}{\text { Tnom }}+\frac{E_{g}(\text { Tnom })}{K \text { Tnom }^{2}}\right)\right] } \\
&=-\left[1-\frac{T}{\text { Tnom }}-\frac{\left.\frac{3 K}{q}(\text { Tnom }-T)+\frac{E_{g}(\text { Tnom })}{\text { Tnom }}(\text { Tnom }-T)\right]}{\Phi_{i}(\text { Tnom })}\right] \\
&=-1+\frac{\Phi_{i}(\text { Tnom })\left(\frac{T}{\text { Tnom }}\right)-\frac{3 K T}{q}\left(1-\frac{\text { Tnom }}{T}\right)}{\Phi_{i}(\text { Tnom })} \\
&+\frac{E_{g}(\text { Tnom })-E_{g}(\text { Tnom })\left(\frac{\text { Tnom }}{T}\right)}{\Phi_{i}(\text { Tnom })}
\end{aligned}
$$

By $1-\frac{\text { Tnom }}{T} \cong \ln \left(\frac{\text { Tnom }}{T}\right)$

$$
\begin{aligned}
\gamma_{T}^{\Phi_{i}(T-\text { Tnom })=-1}+\frac{\Phi_{i}(\text { Tnom })\left(\frac{T}{\text { Tnom }}\right)-3 V_{t} \times \ln \left(\frac{\text { Tnom }}{T}\right)}{\Phi_{i}(\text { Tnom })} \\
+\frac{E_{g}(\text { Tnom })-E_{g}(\text { Tnom })\left(\frac{\text { Tnom }}{T}\right)}{\Phi_{i}(\text { Tnom })}
\end{aligned}
$$

Let $e g=E_{g}($ Tnom $)$, which is the first $E_{g}($ Tnom) of numerator in last term.

This is the third assumption: $e g=E_{g}($ Tnom $)$.

Then all numerators in the right-hand side can be represented by $\Phi_{i}(T)$.

Equation (3.4.15) is rewritten as follows,

$$
\gamma_{T}^{\Phi_{i}}(T-T \text { nom })=-\left(1-\frac{\Phi_{i}(T)}{\Phi_{i}(\text { Tnom })}\right)
$$

Replacement of equation (3.4.16) into equation (3.4.14) yields 


$$
\begin{aligned}
\frac{C_{j}(T)}{C_{j}(\text { Tnom })} & =1+m\left[2 \gamma_{T}^{\varepsilon}(T-\text { Tnom })+\left(1-\frac{\Phi_{i}(T)}{\Phi_{i}(\text { Tnom })}\right)\right] \\
& =1+m\left[0.0004(T-\text { Tnom })+\left(1-\frac{\Phi_{i}(T)}{\Phi_{i}(\text { Tnom })}\right)\right]
\end{aligned}
$$

At last, equation (3.4.17) is applied to two junctions, which are presented in equations (3.4.1) and (3.4.2).

\section{LEAKAGE SATURATION CURRENT}

The leakage saturation current are represented in two junctions, emitter-base and base-collector. They are shown below.

\section{Present model}

$$
\begin{aligned}
& \text { ise }=I S E \times A R E A \times \exp \left(\frac{E G}{N E V_{t}}(\text { ratio }-1)\right) \times \text { ratio }\left(\frac{X T I}{N E}-X T B\right) \\
& \text { isc }=I S C \times A R E A \times \exp \left(\frac{E G}{N C V_{t}}(\text { ratio }-1)\right) \times \text { ratio }\left(\frac{X T I}{N C}-X T B\right)
\end{aligned}
$$

Input parameters are ISE, ISC, NE, and $N C$.

The default values are: $N E=1.5$ and $N C=2$.

$\underline{\text { Rederived model }}$

$$
\begin{gathered}
\text { ise }=I S E \times A R E A \times\left(\frac{C J E}{c j e}\right)^{2 / N E} \times \tau \text { nalE }-2 / N E \times \text { iseref } \\
\text { isc }=I S C \times A R E A \times\left(\frac{C J C}{c j c}\right)^{2 / N C} \times \tau \text { nalC }-2 / N C \times \text { iscref } \\
\text { iseref }=\text { ratio } 3 \times T V / 2 N E \times\left[1+p \times \frac{2}{N E} \times(T-\text { Tnom })\right] \\
\times \exp \left(\frac{E G}{N E V_{t}}(\text { ratio }-1)\right)
\end{gathered}
$$




$$
\begin{gathered}
\text { iscref }=\text { ratio }^{3 X T V / 2 N C} \times\left[1+p \times \frac{2}{N C} \times(T-\text { Tnom })\right] \\
\times \exp \left(\frac{E G}{N C V_{t}}(\text { ratio }-1)\right)
\end{gathered}
$$

Input parameters are $I S E, I S C, N E, N C, \tau$ nalE, and $\tau$ nalC.

The default values are: $N E=2$ and $N C=2$.

This section discusses different origins of the leakage saturation current used in the two models. The nonideal current has been described in the section of the leakage saturation current in Chapter II. Sah [14] indicated that all three recombination currents are relative to intrinsic carrier concentration, the width of space-charge region, and lifetime. Sah implied that the leakage saturation currents is controlled by the leakage emission coefficient. It is the way to obtain the definition of the leakage saturation current shown in equation (2.5.1) for the rederived model. But from a mathematical perspective, the expression of the base current in the low bias region (nonideal base current) can be obtained by comparing with that in the intermediate region (ideal base current), which is related to the junction saturation current. The relationship between the ideal base current and the collector current can be described by the equation shown as follows,

$$
I_{\text {Bideal }}=\frac{I_{C}}{\beta_{F, R}} \quad \Rightarrow \quad I b_{\text {ideal }}=\frac{I S}{\beta_{F, R}}
$$

where $I b_{\text {ideal }}$ and $I s$ are the values of the ideal base and collector currents at zero applied bias respectively. Since the nonideal base current is controlled by the leakage emission coefficient, $n$, the value of the nonideal base current at zero applied bias can be obtained by the equation shown below,

$$
I b_{\text {nonideal }}=\left(I b_{\text {ideal }}\right)^{n}
$$

where the nonideal base current at zero applied bias is named the leakage saturation current. Through equations (3.5.7) and (3.5.8), the relationship between the leakage saturation current and junction saturation current can be determined. This relationship is described by the equation shown below in reference to equations (3.5.1) and (3.5.2) for 
the present model.

$$
I b_{\text {nonideal }}(T)=\frac{I s^{1 / n}(T)}{\beta_{F, R}(T)}
$$

Therefore, the equations of the leakage saturation current for the present model were derived from equation (3.5.9).

According to the definition of the leakage saturation current, equation (2.5.1), except for intrinsic carrier concentration, the temperature behavior of the width of spacecharge region and lifetime is different from that of the parameters in equation (3.5.9), such as diffusion coefficients, widths, and storage charges in the base and emitter or collector regions. Based on this comparison of the definition, equations (3.5.1) and (3.5.2) cannot correctly represent the temperature behavior of the leakage saturation current.

Since no expression for life time is applied in equations (3.5.3) and (3.5.4), the values of the normalized life time in these equations are needed to be measured. Only one expression describing the temperature behavior of life time has been derived by Henry et al [40] in their research regarding the capture cross section and recombination at high temperature. Because of lack of empirical support for this expression in the wide temperature range, this expression was not used for life time in this paper. This equation is shown as follows,

$$
\tau(T)=\text { const. } \times m^{* 3 / 2}(T) \times T^{1 / 2} \times \exp \left(\frac{q E_{B}}{K T}\right)
$$

where

$m^{*}$ is the effective electron mass or hole mass and $E_{B}$ is the activation energy.

Roulston et al [15] defined the total lifetime in the device model as the combination of the Shockley-Read-Hall lifetime and the Auger lifetime, which is:

$$
\frac{1}{\tau_{S A}(N, T)}=\frac{1}{\tau^{S H R}}+\frac{1}{\tau^{A U}}
$$

where 


$$
\tau^{S H R}(N, T)=\frac{\tau_{0}(T)}{1+\frac{N}{N_{0}}}, \quad \tau^{A U}=\frac{1}{C_{n, p} N^{2}}
$$

$C_{n p}$ is the Auger coefficient.

Shockley-Read-Hall lifetime is not only doping concentration but also temperature dependent, which was experimentally proven by Hall [41] for majority-carrier lifetime and theoretically by Shockley et al [42] for both majority- and minority-carrier lifetime. At high doping level lifetime is only doping concentration dependent, supported by Dziewior et al [43] for minority-carrier lifetime that the almost the same values for the Auger coefficients are obtained when dopant concentrations are above $2 \times 10^{18} \mathrm{~cm}^{-3}$ for p-type

material and above $6 \times 10^{18} \mathrm{~cm}^{-3}$ for n-type at $77 \mathrm{~K}, 300 \mathrm{~K}$, and $400 \mathrm{~K}$. However,

Huldt et al [44] suggested that the Auger coefficients are temperature dependent in high doping level within the range of 195 to $372 \mathrm{~K}$. This demonstration was supported by Vaitkus et al [45] that the Auger coefficient is weak temperature dependent when dopant concentration is larger than approximate $10^{18} \mathrm{~cm}^{-3}$ in the range of 100 to $300 \mathrm{~K}$. Since the leakage saturation current is due to recombinations in the space-charge region and the effect of high doping level is not considered in the space-charge region, the Auger lifetime is not included in the rederived model.

If the temperature behavior of the Schockley-Read-Hall lifetime can be represented by the theoretical equation (3.5.10), the normalized lifetime in equation (2.5.7) can be regained by substitution of equation (3.5.10) into (2.5.7). The normalized lifetime is rewritten as follows,

$$
\left[\frac{\tau_{0}(T)}{\tau_{0}(\text { Tnom })}\right]^{-1}=\left(\frac{m_{c, v}(T)}{m_{c, v}(\text { Tnom })}\right)^{-3 / 2} \times\left(\frac{T}{\text { Tnom }}\right)^{-1 / 2} \times \exp \left(\frac{E_{B}}{V_{t}}(\text { ratio }-1)\right)
$$

Replacement of equation (3.5.13) into equation (2.5.11) yields

$$
\frac{I_{0}(T)}{I_{0}(\text { Tnom })}=\left(\frac{M E T}{M H T}\right)^{3 / 2 n} \times \text { ratio }^{2 / n} \times\left(\frac{C_{j 0}(T)}{C_{j 0}(\text { Tnom })}\right)^{-2 / n}
$$




$$
\times \exp \left(p \times \frac{2}{n} \times(T-T \text { nom })\right) \times \exp \left(\frac{E G+2 E_{B}}{n V_{t}}(\text { ratio }-1)\right)
$$

where

The activation energy typically is $0.1 \mathrm{eV}$ for silicon.

Then, equation (3.5.14) can be applied to the emitter-base and the base-collector junctions.

\section{CHARGE-CONTROL MODEL}

\section{Present model}

$$
\begin{aligned}
& o q_{1}=1-\text { ovar } \times \text { vbe }- \text { ovaf } \times v b c \\
& \text { ovar }=\frac{1}{V A R}, \text { ovaf }=\frac{1}{V A F} \\
& V A R=\frac{Q_{B 0}}{\bar{C}_{j e}}, V A F=\frac{Q_{B 0}}{\bar{C}_{j c}} \frac{A_{C}}{A_{E}}
\end{aligned}
$$

Input parameters are $V A R$ and $V A F$.

The default values are: $V A R=$ infinity and $V A F=$ infinity.

$\underline{\text { Rederived model }}$

$$
\begin{aligned}
& o q_{1}=1-\frac{V_{B E}}{\left|\frac{Q_{B 0}}{\text { cjeval }}\right|}-\frac{V_{B C}}{\left|\frac{Q_{B 0}}{\text { cjcval }}\right| \times A C} \\
& \text { cjeval }=\frac{\text { SpiceDepletionCharge }\left(V_{B E},\right. \text { cje, vje, MJE, FC) }}{V_{B E}} \\
& \text { cjcval }=\frac{\text { SpiceDepletionCharge }\left(V_{B C},\right. \text { cjc, vjc, MJC,FC) }}{V_{B C}}
\end{aligned}
$$

Input parameters are $Q_{B 0}$ and $A C$.

The difference between the two models is that the capacitances in equation (3.6.3) 
are constant and in equation (3.6.4) are varied with the applied voltage. Equation (3.6.3) presents the behavior of base-width modulation, which is called the Early voltage. The behavior of the Early voltage is voltage dependent. Therefore, the capacitances in equation (3.6.3) should be allowed to vary with voltages. It is worthwhile to obtain the capacitances dependence of voltage even if the program becomes more complicated. It is a way for the device model to represent this second-order effect for a practical transistor.

So far, the temperature characteristics of effective mass, mobility, energy gap, bandgap narrowing, dielectric constant, and lifetime as used in the device equations have been discussed. The discrepancies between the present model and the rederived model have been described. The base-width modulation in the section of charge-control model has been modified. In the following section, the comparisons of temperature behavior of two models will be presented.

\section{COMPARISON}

In this section all the device equations in the present model will be compared with those in the rederived model under the same doping concentrations and temperature range of 250 to $500 \mathrm{~K}$. All the device equations are normalized, such as $i s / I S, b f / B F$.

\section{Junction saturation current}

Two models have the same equation for the junction saturation current but with different default values of $E G$ and XTI. In the present model the nominal temperature is fixed at $300 \mathrm{~K}$. If the nominal temperature is taken at other temperatures, the junction saturation current in the present model can not provide the correct values. Other device equations have the same shortcoming in the fixed nominal temperature. Here, the junction saturation current is used as an example. In order to show this shortcoming in the present model, the same values of $E G$ and $X T I$ are used in the two models and the nominal temperature is taken at $350 \mathrm{~K}$. The comparison between the two models is shown in Figure 8. The present model is represented by is 1 and is 2 for the rederived model. Since there are different temperature behaviors between electron mobilities and hole mobilities at 
the same doping concentration, the rederived model is 2 can present the different temperature behaviors in an npn and a pnp transistors. The differences between the two transistors are shown in Figure 9 although the differences are small.

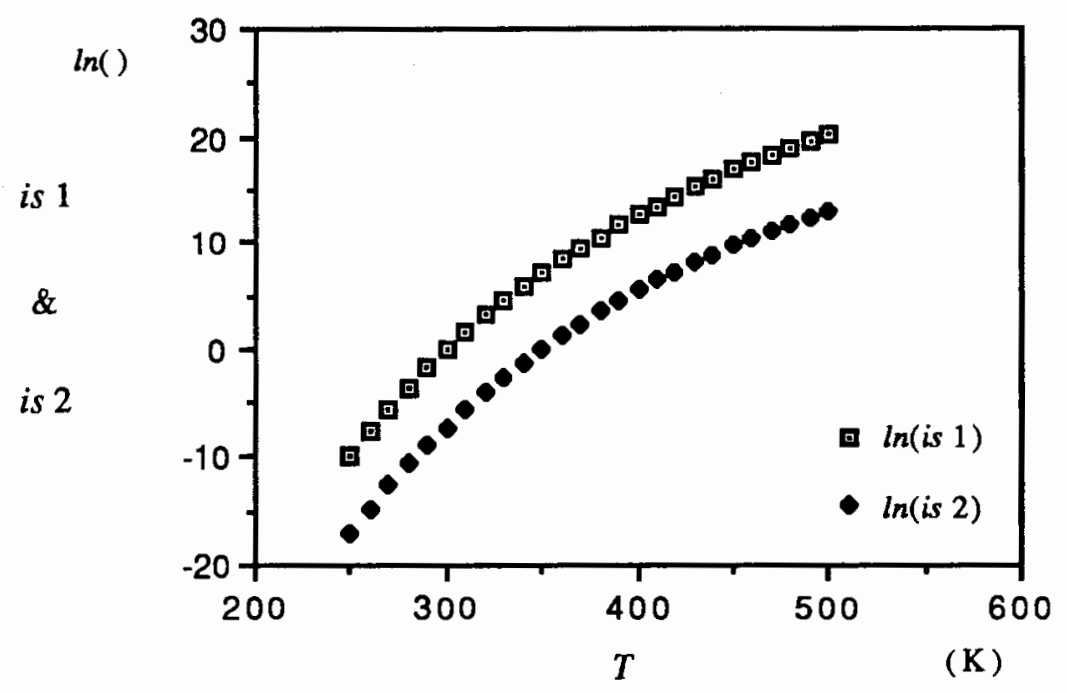

Figure 8. Comparison of is 1 with is 2

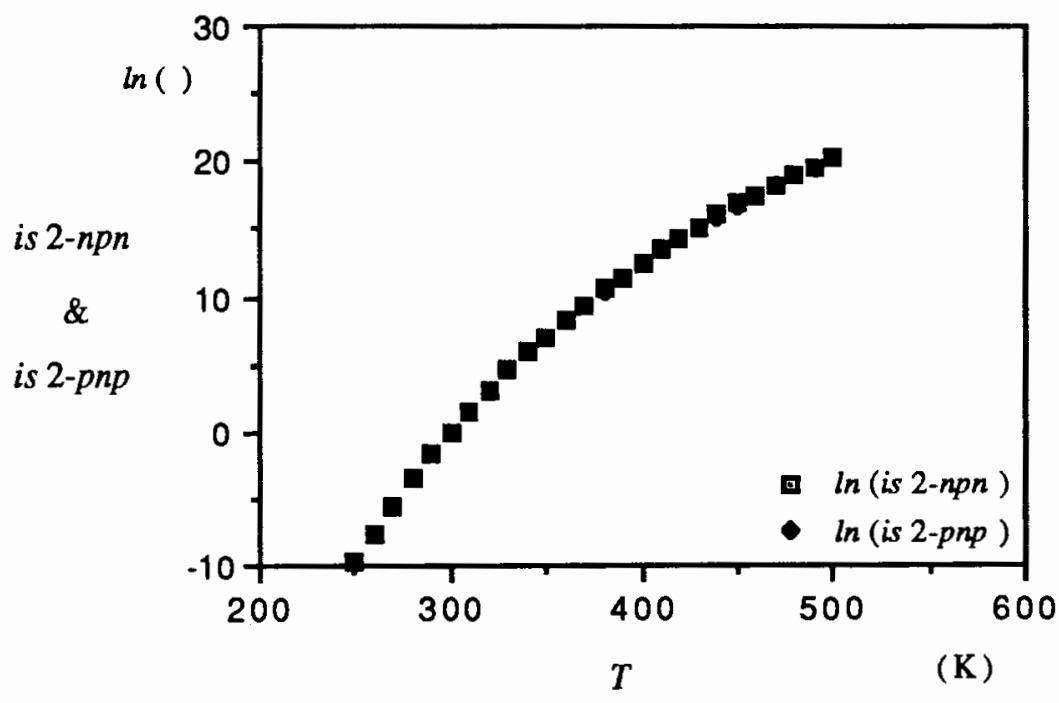

Figure 9. Comparison of an npn transistor with a pnp transistor for is 2. 


\section{Ideal current gain}

The present model is represented by $b f 1$ and $b r l$ for the forward and reverse current gains respectively. The rederived model is represented by $b f 2$ and $b r 2$ for the forward and reverse current gains respectively. The default value for $X T B$ is 1.85 . The comparison of $b f 1$ with $b f 2$ is shown in Figure 10 and for $b r 1$ with $b r 2$ is in Figure 11. The comparison of an npn transistor with a pnp transistor for the rederived model $b f 2$ is shown in Figure 12 and for $b r 2$ is in Figure 13. The nominal temperature of two models is taken at $300 \mathrm{~K}$.

Since the values in the rederived model are mainly determined by the exponential term, which is caused by bandgap narrowing effect, the significant difference between $b f 2$ and $b r 2$ can be seen by comparing Figures 10 and 11 . This behavior can not be shown in $b f 1$ and $b r 1$ in the present model since the same $X T B$ is used. The differences between an npn and a pnp transistors for reverse current gain are more than for forward current gain since the values between $X T B F$ and $X T B R$ are quite different.

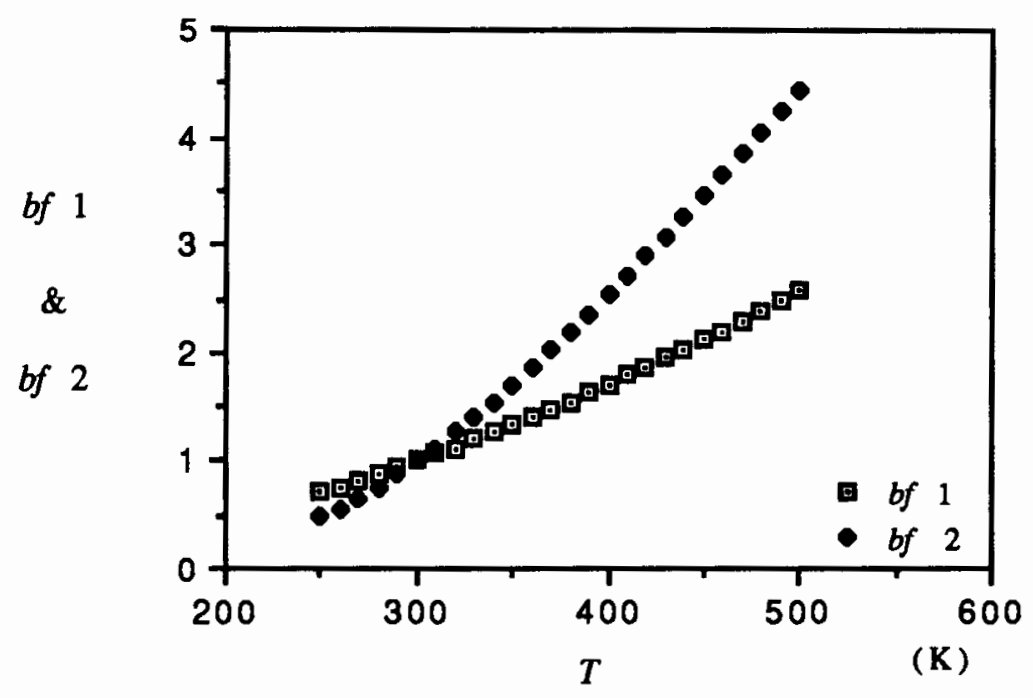

Figure 10. Comparison of $b f 1$ with $b f 2$. 


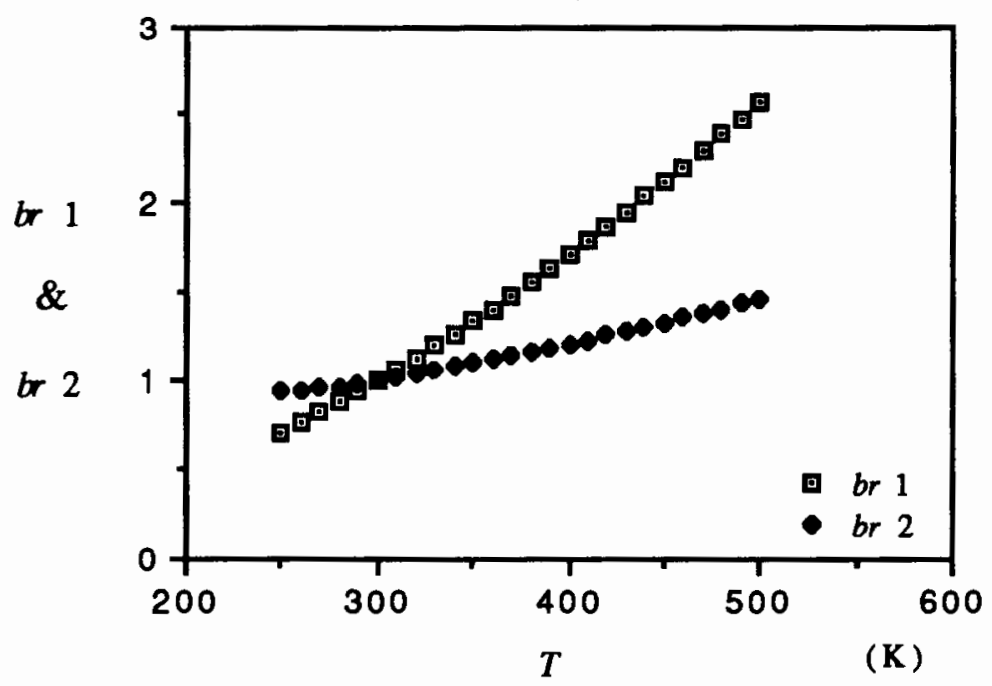

Figure 11. Comparison of $b r 1$ with $b r 2$.

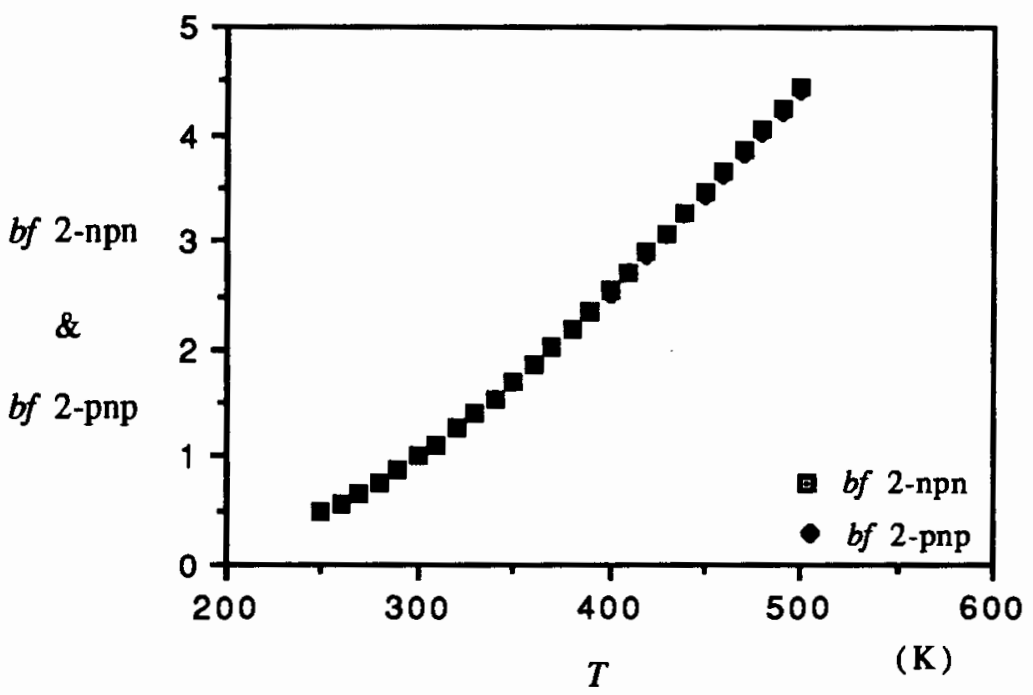

Eigure 12. Comparison of an npn transistor with a pnp transistor for $b f 2$. 


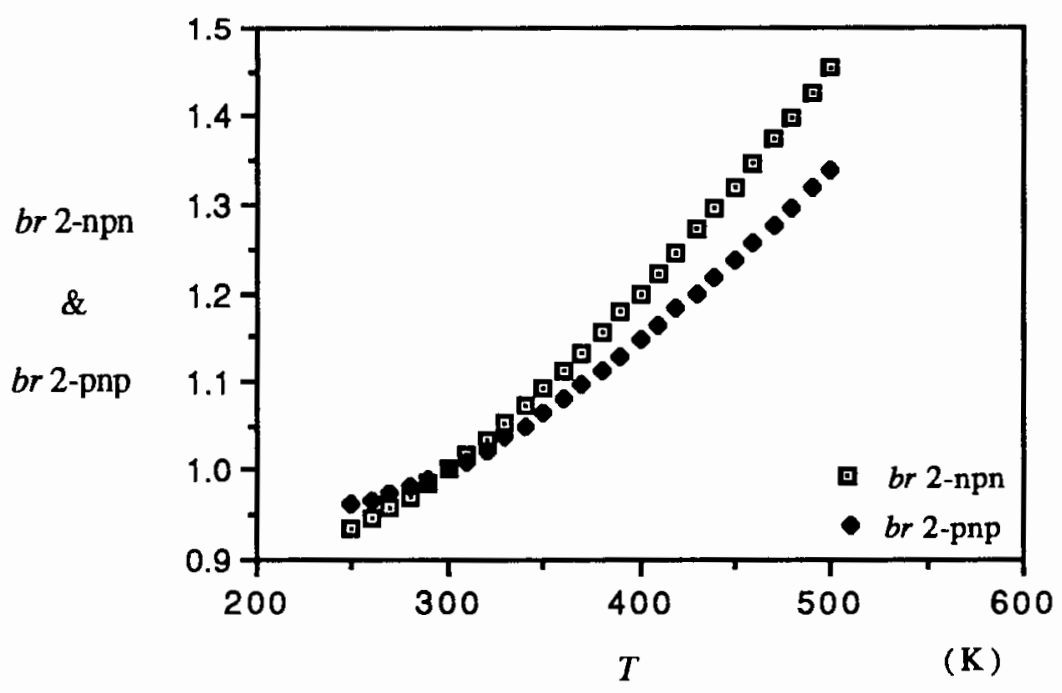

Figure 13. Comparison of an npn transistor with a pnp transistor for $b r 2$.

\section{Built-in junction potential}

The present model is represented by vje 1 and $v j c 1$. The rederived model is represented by $v j e 2$ and $v j c 2$. Since the values from $v j e 1$ and $v j c 1$ are close to those from $v j e 2$ and $v j c 2$ respectively, only the comparison of vje 1 with vje 2 will be shown in Figure 14. The nominal temperature of two models is taken at $300 \mathrm{~K}$. To explain the limitation of hardware numbers in the present model, the different energy gap of other semiconductors, rather than silicon, is used in the built-in junction potential for two models. The energy gap of GaAs is used. Since those hardware numbers, the built-in junction potential in the present model always keeps the same value which is only valid for silicon. The error made by the present model vje 1 and the correct values provided by the rederived model vje2 are shown in Figure 15. 


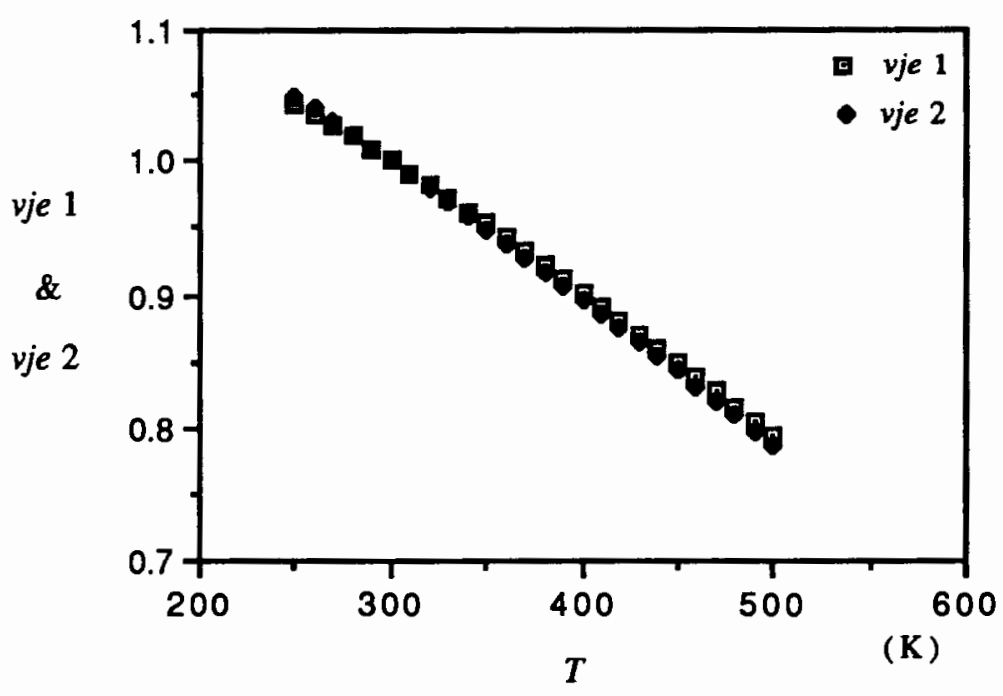

Figure 14. Comparison of vje1 with vje2.

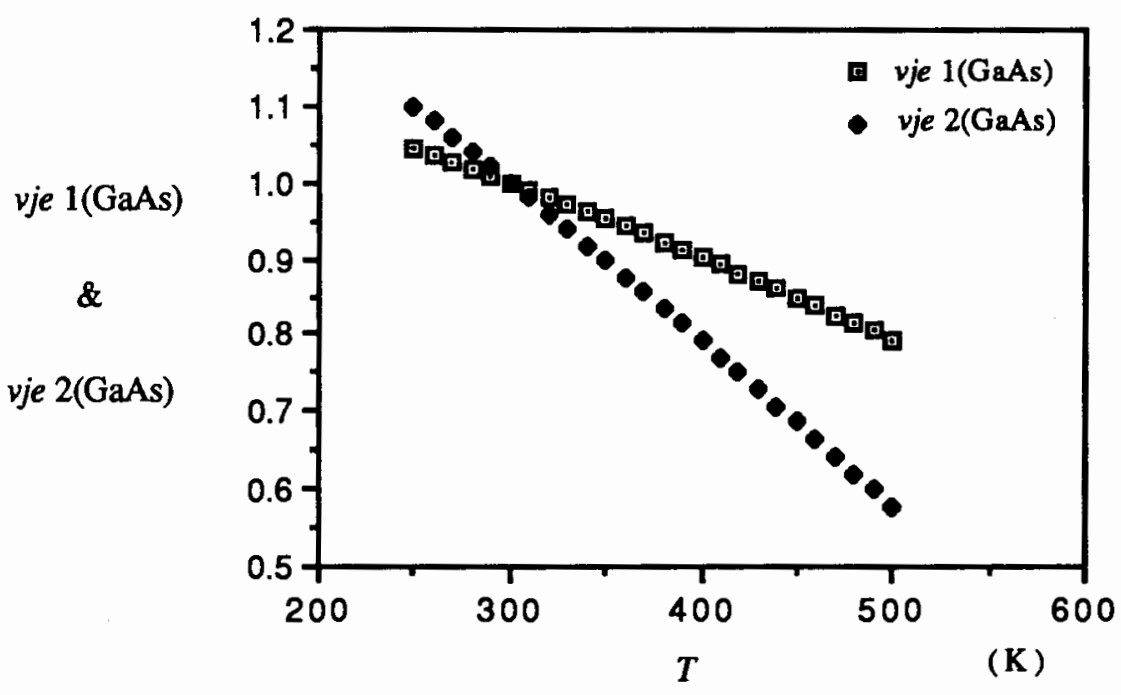

Figure 15. Comparison of vje 1 with vje2 in GaAs.

Zero-bias junction capacitance

The present model is represented by cjel and cjc1. The rederived model is represented by $c j e 2$ and $c j c 2$. Since the values from $c j e 1$ and $c j c 1$ are close to those from cje 2 and $c j c 2$ respectively, only the comparison of $c j e 1$ with cje 2 will be shown in Figure 
16. The nominal temperature of two models is taken at $300 \mathrm{~K}$. Although the zero-bias junction capacitance of the present model was derived under three assumptions, similar values with those from the rederived model are obtained for the present model. This result is shown in Figure 16.

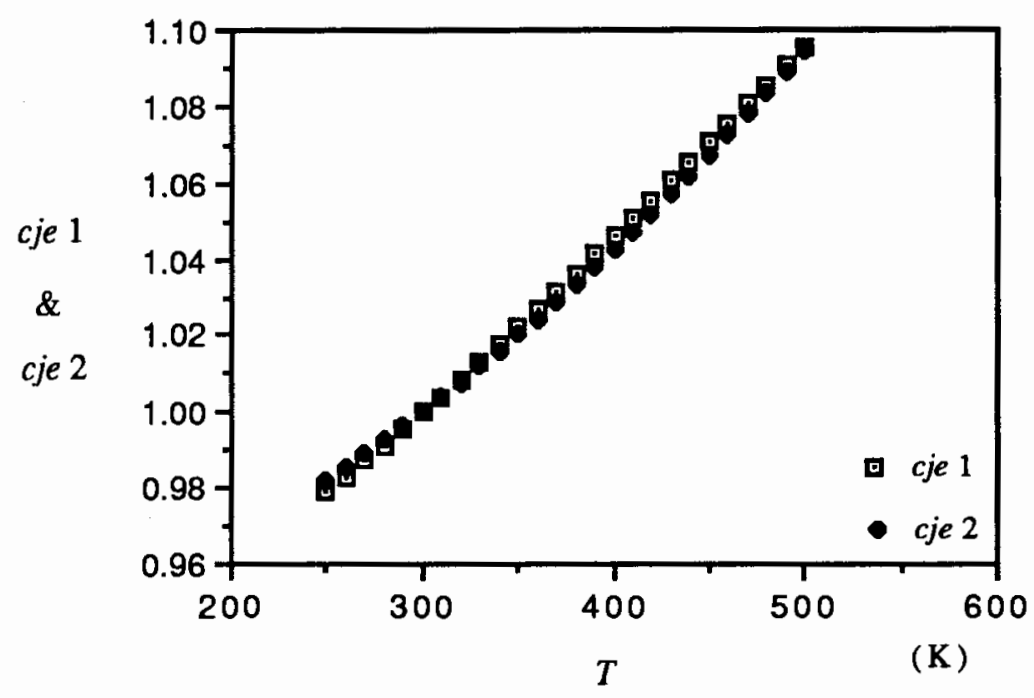

Figure 16. Comparison of cje1 with cje2.

\section{Leakage saturation current}

Although the rederived model can correctly present temperature behavior of the leakage saturation current, its equations shown in (3.5.3-6) are more complicated than equations of the present model. The rederived model dose not seem to be more effective. To find a simpler model which can correctly present the temperature behavior of the leakage saturation current is not an easy task, which is in need in the further. Here, the comparison between the two models is to show how different the values between the two models are. The present model is represented by ise 1 and isc 1 . The rederived model is represented by ise 2 and isc 2 under the assumption that the normalized lifetime equals constant, i.e., the lifetime is temperature independent. At the same time, the rederived model which includes theoretical lifetime as a function of temperature is represented by ise2-tnalE and isc2-tnalC. Since the similar temperature behavior can be obtained in 
comparison between ise 1 with ise 2 and isc 1 with isc 2 , only the comparison of ise 1 with ise 2 is shown in Figure 17, as well as ise 1 with ise 2-tnalE. The difference in values between ise 1 and ise 2 is as great as $0.3-10$ times in the range of $[250,500] \mathrm{K}$ and $0.6-3$ times between ise 1 and ise2-tnalE.

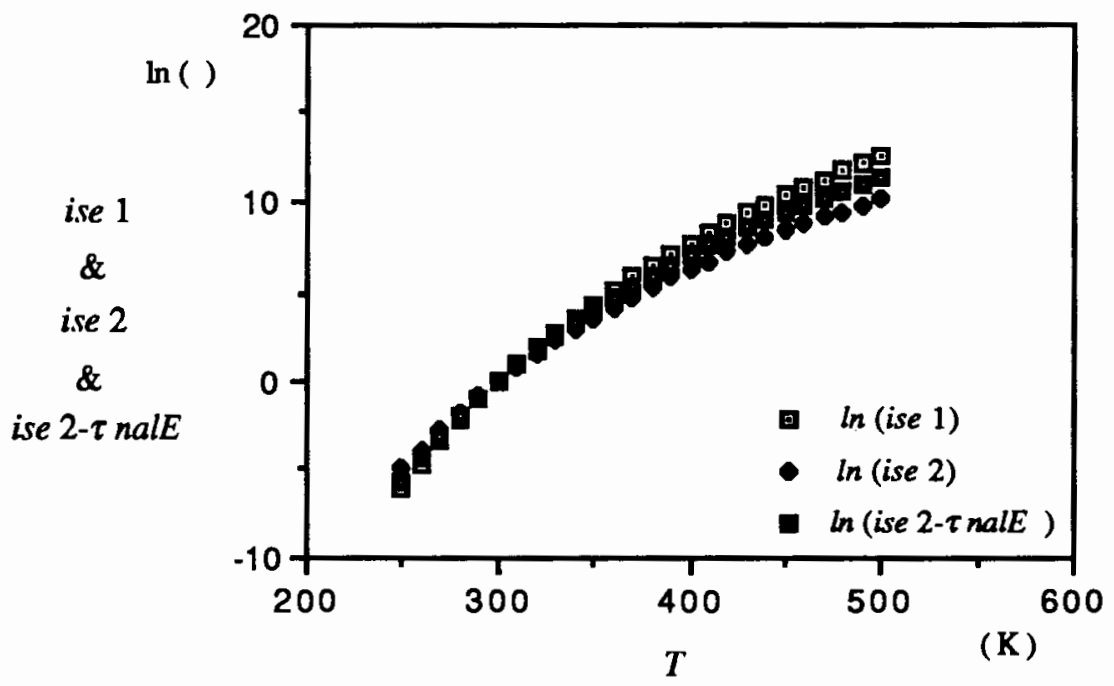

Figure 17. Comparison among ise1, ise2, and ise2 with the theoretical lifetime. 


\section{CHAPTER IV}

\section{CONCLUSION}

The derivations and applications of expressions of the specific temperature-related parameters and Early effect in the charge-control model have been presented in Chapter II. Some parameters dependence of temperature, doping concentration, or presence of two effects have been introduced in the rederived model resulting in different temperature behavior from the present model. The temperature effects of these parameters have been discussed in Chapter III and the comparisons of the specific parameters between two models have also been presented.

Complicated equations of mobility and effective mass have been represented by the simplified equations so that the temperature behavior of mobility and effective mass can be introduced in the specific parameters. Due to different temperature behaviors between electron-carrier and hole-carrier mobilities, the coefficients XTUn and XTUp provide a way for the rederived model to distinguish the temperature behavior of an npn transistor from that of a pnp transistor, which the present model is incapable of performing. The inclusion of the effects of bandgap narrowing, temperature-dependent mobility, and effective mass has made the rederived model more accurate, but, on the other hand, the number of input parameters has been increased by nine and the expressions have made the GP2 program more complex. However, in recent years the speed of computers has been dramatically improved, which allows more accurate models to run using a relatively short period of time.

Rederived model can still be improved. Further work will be needed to obtain empirical expressions for the minority-carrier mobilities and the lifetime. 


\section{REFERENCES}

[1] I. Getreu, Modeling the Bipolar Transistor, Tektronix, Inc., Beaverton, Oregon (1976).

[2] H. K. Gummel and H. C. Poon, "An Integral Charge Control Model of Bipolar Transistors," The Bell System Technical Journal, vol. 49, pp. 827-852 (May-June 1970).

[3] EE Simulation Group, TEKSPICE Reference Manual - Version IE, CAX Center, Tektronix, Inc., Beaverton, Oregon (1987).

[4] R. S. Muller and T. I. Kamins, Device Electronics for Integrated Circuit, 2d ed., John Wiley \& Sons, New York (1986).

[5] H. D. Barber, "Effective Mass and Intrinsic Concentration in Silicon," SolidState Electronics, Vl. 10, pp. 1039-1051 (1967).

[6] F. H. Gaensslen and R. C. Jaeger, "Temperature Dependent Threshold Behavior of Depletion Mode MOSFETs - Characterization and Simulation," Solid-State Electronics, Vol. 22, pp. 423-430 (1979).

[7] J. W. Slotboom and H. C. De Graaff, "Measurements of Bandgap Narrowing in in Si Bipolar Transistors," Solid-State Electronics, Vol. 19, pp. 857-862 (1976).

[8] N. D. Arora, J. R. Hauser, and D. J. Roulston, "Electron and Hole Mobilities in Silicon as a Function of Concentration and Temperature," IEEE Transactions on Electron Devices, Vol. ED-29, No. 2, pp. 292-295 (Feb. 1982).

[9] R. P. Mertens, H. J. DeMan, and R. J. Van Overstraeten, "Calculation of the Emitter Efficiency of Bipolar Transistors,"IEEE Transactions on Electron Devices, Vol. ED-20, No. 9, pp. $772-778$ (Sept. 1973).

[10] D. J. Roulston, Bipolar Semiconductor Devices, McGraw-Hill, Inc. (1990).

[11] M. Cardona, W. Paul, and H. Brooks, "Dielectric Constant of Germanium and Silicon as a function of Volume,"J. Phys. Chem. Solids, Vbl. 8, pp. 204-206 (1959).

[12] P. Ashburn, D. V. Morgan, and M. J. Howes, "A Theoretical and Experimental Study of Recombination in Silicon p-n Junctions," Solid-State Electronics, Vol. 18, pp. 569-577 (1975).

[13] C. T. Sah, "Effects of Electrons and Holes on the Transition Layer Characteristics of Linearly Graded P-N Junctions," Proceeding of the IRE, Vol. 49, pp. 603-618 (March 1961). 
[14] C. T. Sah, "Effect of Surface Recombination and Channel on P-N Junction and Transistor Characteristics," IRE Transactions on Electron Devices, Vol. ED-9, pp. 94-108 (Jan. 1962).

[15] D. J. Roulston, N. D. Arora, and S. G. Chamberlain, "Modeling and Measurement of Minority-Carrier Lifetime versus Doping in Diffused Layers of $\mathrm{n}^{+}$-p Silicon Diodes," IEEE Transactions on Electron Devices, Vol. ED-29, No. 2, pp. 284-291 (Feb. 1982).

[16] Y. P. Varshni, "Temperature Dependence of the Energy Gap in Semiconductors," Physica, Vol. 34, pp. 149-154 (1967).

[17] C. D. Thurmond, "The Standard Thermodynamic Functions for the Formation of Electrons and Holes in Ge, Si, GaAs, and GaP," J. Electrochem. Soc.: SolidState Science and Technology, Vol. 122, No. 8, pp. 1133-1141 (Aug. 1975).

[18] W. Bludau, A. Onton, and W. Heinke, "Temperature Dependence of the Band Gap of Silicon," Journal of Applied Physics, Vol. 45, No. 4, pp. 1846-1848 (April 1974).

[19] K. L. Shaklee and R. E. Nahory, Physical Review Letters, Vol. 24, pp. 942-945 (1970).

[20] A. Neugroschel, S. C. Pao, and F. A. Lindholm, "A Method for Determining Energy Gap Narrowing in Highly Doped Semiconductors," IEEE Transactions on Electron Devices, Vol. ED-29, No. 5, pp. 894-902 (May 1982).

[21] D. Dale Kleppinger and F. A. Lindholm, "Impurity Concentration Dependent Density of States and Resulting Fermi Level for Silicon," Solid-State Electronics, Vol. 14, pp. 407-416 (1971).

[22] D. S. Lee and J. G. Fossum, "Energy-Band Distortion in Highly Doped Silicon," IEEE Transactions on Electron Devices, Vi. ED-30, No. 6, pp. 626-634 (June 1983).

[23] W. L. Kauffman and A. A. Bergh, "The Temperature Dependence of Ideal Gain in Double Diffused Silicon Transistors," IEEE Transactions on Electron Devices, Vol. ED-15, No.10, pp. $732-735$ (Oct. 1968).

[24] H. S. Bennett, "Heavy Doping Effects on Bandgaps, Effective Intrinsic Carrier Concentrations and Carrier Mobilities and Lifetimes," Solid-State Electronics, Vol. 28, Nos. 1 / 2, pp. 193-200 (1985).

[25] A. W. Wieder, "Emitter Effects in Shallow Bipolar Devices: Measurements and Consequences," IEEE Transactions on Electron Devices, Vol. ED-27, No. 8, pp. 1402-1408 (Aug. 1980).

[26] R. P. Mertens, J. L. Van Meerbergen, J. F. Nijs, and R. J. Van Overstraeten, "Measurement of the Minority-Carrier Transport Parameters in Heavily Doped Silicon," IEEE Transactions on Electron Devices, Vol. ED-27, No. 5, pp. 949-955 (May 1980). 
[27] G. E. Possin, M. S. Adler, B. J. Baliga, "Measurements of the p-n Product in Heavily Doped Epitaxial Emitters," IEEE Transactions on Electron Devices, Vol. ED-31, No. 1, pp. 3-17 (Jan. 1984).

[28] W. P. Dumke, "Comparison of Band-Gap Shrinkage Observed in Luminescence from $\mathrm{n}^{+}-\mathrm{Si}$ with That from Transport and Optical Absorption measurements," Applied Physics Letters, Vol. 42, No. 2, pp. 196-198 (Jan. 15 1983).

[29] J. G. Fossum, D. E. Burk, and S. -Y. Yung, "Effective Minority-Carrier Mobility in Heavily Doped Silicon Defined by Trapping and Energy-Gap Narrowing," IEEE Transactions on Electron Devices, Vbl. ED-32, No. 9, pp. 1874-1877 (Sept. 1985).

[30] J. Dziewior and D. Silber, "Minority-Carrier Diffusion Coefficients in Highly Doped Silicon," Applied Physics Letters, Vol. 35, No. 2, pp. 170-172 (July 15 1979).

[31] D. E. Burk and V. De La Torre, "An Empirical Fit To Minority Hole Mobilities," IEEE Electron Device Letters, Vol. EDL-5, No. 7, pp. 231-233 (July 1984).

[32] A. Neugroschel, "Minority-Carrier Diffusion Coefficients and Mobilities in Silicon," IEEE Electron Device Letters, Vol. EDL-6, No. 8, pp. 425-427 (Aug. 1985).

[33] S. E. Swirhun, Y. -H. Kwark and R. M. Swanson, "Measurement of Electron Lifetime, Electron Mobility and Band-Gap Narrowing in Heavily Doped p-Type Silicon," IEDM Tech. Dig., pp. 24-27 (1986).

[34] A. Neugroschel and F. A. Lindholm, "Evidence for Low Diffusivity and Mobility of Minority Carriers in Highly Doped Si and Interpretation," Applied Physics Letters, Vbl. 42, No. 2, pp. 176-178 (Jan. 15 1983).

[35] S. - Y. Yung, D. E. Burk, and J. G. Fossum, "Numerical Simulation of Temperature-Dependent Minority-Hole Transport in $\mathrm{n}^{+}$Silicon Emitters," Solid State Electronics, V61. 29, No. 12, pp. 1243-1251 (1986).

[36] J. del Alamo, S. Swirhun, and R. M. Swanson, "Simultaneous Measurement of Hole Lifetime, Hole Mobility and Bandgap Narrowing in Heavily Doped n-Type Silicon,"IEDM Tech. Dig., pp. 290-293 (1985).

[37] T. E. Idleman, F. S. Jenkins, W. J. McCalla, and D. O. Pederson, "SLIC-A Simulator for Linear Integrated Circuits," IEEE Journal of Solid-State Circuits, Vol. SC-6, No. 4, pp. 188-203 (Aug. 1971).

[38] H. -M. Rein, H. v. Rohr, and P. Wennekers, "A Contribution to the Current Gain Temperature Dependence of Bipolar Transistors," Solid-State Electronics, Vol. 21, pp. 439-442 (1978). 
[39] H. K. Gummel and D. L. Scharfetter, "Depletion-Layer Capacitance of $\mathrm{p}^{+} \mathrm{n}$ Step Junctions," Journal of Applied Physics, Vol. 38, No. 5, pp. 2148-2153 (April 1967).

[40] C. H. Henry and D. V. Lang, "Nonradiative Capture and Recombination by Multiphonon Emission in GaAs and GaP," Physical Review B, Vol. 15, No. 2, pp. 989-1016 (Jan. 15 1977).

[41] R. N. Hall, "Electron-Hole Recombination in Germanium," Physical Review, Vol. 87, p. 387 (July-Sept. 1952).

[42] W. Shockley and W. T. Read, Jr., "Statistics of the Recombinations of Holes and Electrons," Physical Review, Vol. 87, No. 5, pp. 835-842 (Sept. 1 1952).

[43] J. Dziewior and W. Schmid, "Auger Coefficients for Highly Doped and Highly Excited Silicon," Applied Physics Letters, Vol. 31, No. 5, pp. 346-348 (Sept. 1 1977).

[44] L. Huldt, N. G. Nilsson, and K. G. Svantesson, "The Temperature Dependence of Band-to Band Auger Recombination in Silicon," Applied Physics Letters, Vol. 35, No. 10, pp. 776-777 (Nov. 15 1979).

[45] Yu. Vaitkus and V. Grivitskas, "Dependence of the Rate of Interband Auger Recombination on the Carrier Density in Silicon," Sov. Phys. Semicond., Vol. 15, No. 10, pp. 1102-1108 (Oct. 1981). 